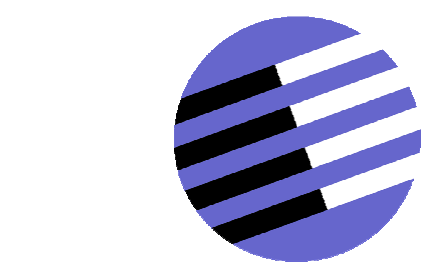

GOVERNANCE AND THE EFFICIENCY

OF ECONOMIC SYSTEMS

GES Y

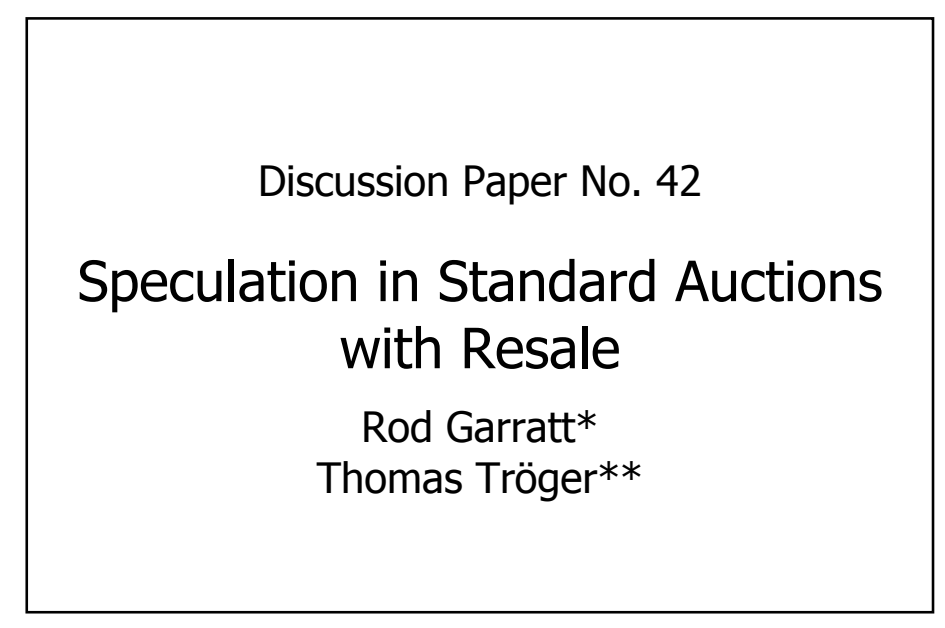

May 2005

*Rod Garratt, Department of Economics, University of California Santa Barbara, CA 93106, USA

**Thomas Tröger, Department of Economics, University of Bonn, Adenauerallee 24-42, D-53113 Bonn, Germany, ttroeger@uni-bonn.de

Financial support from the Deutsche Forschungsgemeinschaft through SFB/TR 15 is gratefully acknowledged.

Sonderforschungsbereich/Transregio $15 \cdot$ www.gesy.uni-mannheim.de

Universität Mannheim · Freie Universität Berlin · Humboldt-Universität zu Berlin · Ludwig-Maximilians-Universität München Rheinische Friedrich-Wilhelms-Universität Bonn · Zentrum für Europäische Wirtschaftsforschung Mannheim 


\title{
Speculation in Standard Auctions with Resale
}

\author{
By Rod Garratt and Thomas Tröger ${ }^{1}$
}

May 4, 2005

\begin{abstract}
In standard auctions with symmetric, independent private value bidders resale creates a role for a speculator - a bidder who is commonly known to have no use value for the good on sale. For second-price and English auctions the efficient value-bidding equilibrium coexists with a continuum of inefficient equilibria in which the speculator wins the auction and makes positive profits. First-price and Dutch auctions have an essentially unique equilibrium, and whether or not the speculator wins the auction and distorts the final allocation depends on the number of bidders, the value distribution, and the discount factor. Speculators do not make profits in first-price or Dutch auctions.
\end{abstract}

KEYWORDS: standard auctions, speculation, resale, efficiency JEL CODE: D44

\footnotetext{
${ }^{1}$ This paper and its online supplement include content from two previous working papers: "Speculation in Second-Price Auctions With Resale," 2003, by Garratt and Tröger, and "Speculations in First-Price Auctions With Resale," 2003, by Tröger. We are grateful to Ted Bergstrom, Ken Binmore, James McAndrews, Eddie Dekel, David Easley, Gunnar Gunnarson, Matthew Jackson, Philippe Jehiel, Alexander Koch, Stephan Lauermann, Steve LeRoy, Benny Moldovanu, Georg Nöldeke, Jörg Oechssler, Thomas Palfrey, Hugo Sonnenschein, Ennio Stacchetti, Bill Zame, Charles Zheng, and three anonymous referees for helpful comments. Financial Support by the German Science Foundation (DFG) through SFB/TR 15 "Governance and the Efficiency of Economic Systems" is gratefully acknowledged.
} 


\section{Introduction}

Standard auctions - open English, sealed-bid first-price, sealed-bid second-price and Dutchare used in many instances where resale is permitted. For instance, a good purchased on eBay can be resold on eBay. Nevertheless, much of the received theory of standard auctions assumes that resale is not possible (cf. Klemperer's 1999 literature survey). In environments where any standard auction has an efficient equilibrium, so that no obvious motive for resale exists, it is tempting to think that the absence of resale opportunities is an inconsequential assumption. However, the assumption still matters.

This paper considers an environment with symmetric independent private value (SIPV) bidders and an additional bidder, called speculator, who is commonly known to have no use value for the good on sale. In the absence of a resale opportunity abstaining is, of course, a dominant strategy for the speculator, and any standard auction allocates the good efficiently and yields identical revenues (Vickrey, 1961). We show that if an inter-bidder resale opportunity exists, the speculator can play an active role in any standard auction format. If she is active, the final allocation is not efficient, and there is no longer allocation or revenue equivalence across standard auctions.

The main insights are conveyed using just two bidders: a single bidder with a private use value for the good and a speculator. The bidders engage in a 2-period interaction where first a standard auction takes place and then the winner can make a take-it-or-leave-it offer to the loser. We construct perfect Bayesian equilibria in undominated strategies. The equilibrium constructions generalize, under appropriate assumptions, to environments with any number of SIPV bidders (see Section 5). Moreover, the equilibria remain valid if any number of speculators can bid in the auction (see REMARK 3 and REMARK 7).

In the case of first-price auctions we assume that, as in a Dutch auction, the loser's bid remains private. There exists an essentially unique equilibrium. ${ }^{1}$ The private-value bidder uses a strictly increasing bid function and the speculator randomizes her bid in such a way

\footnotetext{
${ }^{1}$ The only possible source of multiplicity is that the speculator's post-auction belief may allow for multiple optimal resale offers; but this happens with probability 0 on the equilibrium path.
} 
that she may tie with any type of the private-value bidder. The speculator makes zero profit in equilibrium: the larger her realized bid the larger her resale profit when she wins, but her bid always equals her resale profit.

For second-price or English auctions there is a continuum of equilibria that differ by allocation and profitability to the speculator. For each use value in the support of the underlying probability distribution, there exists an equilibrium where all private-value bidder types having lower use values abstain from the auction and wait for a resale offer from the speculator while types having higher use values bid them and win the auction. Unlike in a first-price or Dutch auction, a speculator can make profits in a second-price or English auction. The larger the chosen use value, the larger the speculator's equilibrium resale offer and payoff.

Previous work on standard auctions with resale considers environments where resale is required to achieve an efficient allocation. ${ }^{2}$ Haile $(2000,2003)$ evaluates the properties of standard auctions in symmetric environments where precise information about use values becomes available only after the initial auction. Haile (1999) and Bose and Deltas (2002) consider environments where some bidders cannot participate in the initial auction. ${ }^{3}$ Finally, Gupta and Lebrun (1999) examines a first-price auction with two asymmetric private-value bidders who observe each other's use value after the auction. ${ }^{4}$

Bose and Deltas (2002), as well as Bikhchandani and Huang (1989) in a multiple-unit auction setting, appear to be the only auction models apart from ours that explicitly consider 0-value bidders. In both of these models the 0-value bidders represent intermediaries or

\footnotetext{
${ }^{2}$ There is also a growing literature on optimal auctions with resale, see Jehiel an Moldovanu (1999), Ausubel and Crampton (1999), Calzolari and Pavan (2002), and Zheng (2002). Our model is closest to Zheng. He considers general asymmetric environments with independent private values. At any stage, including the initial stage, the current owner of the good chooses a mechanism that is optimal given that each future owner will choose an optimal resale mechanism. Zheng's main contribution is the construction of an equilibrium that implements the initial-seller-optimal allocation via resale under certain assumptions. Speculators are not needed in this construction and it is optimal for each current owner to exclude them.

${ }^{3}$ This motive for resale is proposed in Milgrom (1987).

${ }^{4}$ It is well-known that a first-price auction with asymmetric private-value bidders and no resale opportunity is generally inefficient (see Krishna, 2002, Section 4.3.3).
} 
middlemen in environments like treasury bill auctions or large real-estate auctions where the final consumers are confined to the resale market, while our model addresses how 0-value bidders can "invade" markets with private-value bidders.

Section 2 introduces the model. In Section 3 we construct the equilibrium for first-price and Dutch auctions with resale. Section 4 deals with equilibria for second-price and English auctions with resale, and section 5 discusses the extension of the model to multiple SIPV bidders.

\section{Model}

We consider environments with two risk-neutral bidders who are interested in purchasing a single indivisible private good. Bidder 1 has the random use value $\tilde{\theta}_{1} \in[0,1]$ for the good. Let $F$ denote the distribution function for $\tilde{\theta}_{1}$. We assume that $F$ has a density $f$ that is positive and continuous on $[0,1]$ and is identically 0 elsewhere. Bidder 2 has the commonly known use value $\theta_{2}=0$. We call bidder 2 a speculator.

We consider a 2-period interaction. Before period 1, bidder 1 privately learns the realization of her use value, $\tilde{\theta}_{1}=\theta_{1}$. In period 1 , the good is offered via a sealed-bid first-price auction or second-price auction without reserve price. The highest bidder becomes the new owner of the good. To simplify the presentation we assume that bidder 1 wins all ties. The period-1 winner either consumes the good in period 1 or makes a take-it-or-leave-it offer in period 2; if she fails to resell the good she consumes it in period 2. Period-2 payoffs are discounted according to a common factor $\delta \in(0,1) .^{5}$

Actions taken in period 2 may depend on information that is revealed during period 1 . In the first-price case we assume that the winner's bid becomes public after the auction while the loser's bid remains private. In the second-price auction case, we assume that the loser's bid becomes public while the winner's bid remains private. These bid revelation assumptions

\footnotetext{
${ }^{5}$ The equilibria we construct remain valid without discounting $(\delta=1)$, but it seems worthwhile to emphasize that the constructions are robust to discounting. The only instance where we actually use the discounting assumption $\delta<1$ is in the equilibrium uniqueness proof in the first-price auction setting.
} 
make the first-price auction strategically equivalent to a Dutch auction (which stops at the moment the winner's bid is revealed) and make the second-price auction strategically equivalent to an English auction (which stops at the moment the loser's bid is revealed).

We will say that the speculator plays an active role if she wins the auction in period 1 with positive probability.

\section{First-price/Dutch auctions with resale}

In this section we construct and discuss the perfect Bayesian equilibrium of a first-price auction with resale. Additional notation is required. Let $\beta_{1}$ denote bidder 1's bid as a function of her use value and $H$ denote the probability distribution for bidder 2's (possibly randomized) bid $\tilde{b}_{2}$. The probability distribution $\Pi\left(\cdot \mid b_{2}\right)$ describes bidder 2's post-auction belief about bidder 1's use value if bidder 2 bids $b_{2}$ and wins. $T\left(b_{2}\right)$ denotes bidder 2's resale offer. For all bids $b_{1} \geq 0$ and $\theta_{1} \in[0,1]$, bidder 1 's payoff is

$$
u_{1}\left(b_{1}, \theta_{1}\right)=H(b)\left(\theta_{1}-b_{1}\right)+\delta \int_{\left(b_{1}, \infty\right)} \mathbf{1}_{\theta_{1} \geq T(b)}\left(\theta_{1}-T(b)\right) \mathrm{d} H(b) .
$$

For all bids $b_{2} \geq 0$, bidder 2's payoff is

$$
u_{2}\left(b_{2}\right)=\operatorname{Pr}\left[\beta_{1}\left(\tilde{\theta}_{1}\right)<b_{2}\right]\left(-b_{2}+\left(1-\Pi_{-}\left(T\left(b_{2}\right) \mid b_{2}\right)\right) \delta T\left(b_{2}\right)\right)
$$

where $\Pi_{-}\left(p \mid b_{2}\right)=\lim _{p^{\prime} / p} \Pi\left(p^{\prime} \mid b_{2}\right)$ (for all $p \geq 0$ ) denotes the posterior probability that bidder 1's use value is less than $p$. The perfect Bayesian equilibrium conditions are Bayes rule (3), optimality of the resale offer (4), and optimal bidding $(5,6)$, as shown in the following

Definition 1 A tuple $\left(\beta_{1}, H, T, \Pi\right)$ is a perfect Bayesian equilibrium for the first-price auction with resale if

$$
\begin{aligned}
& \forall \theta_{1} \in[0,1], b_{2} \geq 0: \\
& \Pi\left(\theta_{1} \mid b_{2}\right) \operatorname{Pr}\left[\beta_{1}\left(\tilde{\theta}_{1}\right)<b_{2}\right]=\operatorname{Pr}\left[\tilde{\theta}_{1} \leq \theta_{1}, \beta_{1}\left(\tilde{\theta}_{1}\right)<b_{2}\right], \\
& \forall b_{2} \geq 0: T\left(b_{2}\right) \in \arg \max _{p \geq 0}\left(1-\Pi_{-}\left(p \mid b_{2}\right)\right) p,
\end{aligned}
$$




$$
\begin{gathered}
\forall \theta_{1} \in[0,1]: \beta_{1}\left(\theta_{1}\right) \in \arg \max _{b_{1} \geq 0} u_{1}\left(b_{1}, \theta_{1}\right), \\
\operatorname{Pr}\left[\tilde{b}_{2} \in \arg \max _{b \geq 0} u_{2}(b)\right]=1,
\end{gathered}
$$

where $\tilde{b}_{2}$ is distributed according to $H$.

Proposition 1 describes the equilibrium. Condition (7) says that the bid of any bidder-1 type $\theta_{1}$ equals the discounted profit of a monopolist who faces the bidder- 1 types in $\left[0, \theta_{1}\right]$. Bidder 2 randomizes her bid according to (8), in which the distribution $H$ is constructed such that bidder 1's bid function is optimal. In particular, the support of $H$ equals bidder 1's bid range $\left[0, \beta_{1}(1)\right]{ }^{6}$ The resale offer function (10) follows from the post-auction beliefs (9): if bidder 2 wins then she believes that the highest losing type of bidder 1 is the type that would have resulted in a tie.

Proposition 1 A tuple $\left(\beta_{1}, H, T, \Pi\right)$ is a perfect Bayesian equilibrium for the first-price auction with resale if and only if conditions (7) (8), (9), and (10) hold.

$$
\begin{gathered}
\forall \theta_{1} \in[0,1]: \beta_{1}\left(\theta_{1}\right)=\delta \max _{p \geq 0} \frac{F\left(\theta_{1}\right)-F(p)}{F\left(\theta_{1}\right)} p . \\
\forall b_{2} \in\left(0, \beta_{1}(1)\right]: H\left(b_{2}\right)=e^{-\int_{b_{2}}^{\beta_{1}(1)} \frac{1}{(1-\delta) \phi_{1}(b)-b+\delta T(b)}} d b
\end{gathered}
$$

where $\phi_{1}$ denotes the inverse of $\beta_{1}$.

$$
\forall b_{2} \geq 0, \theta_{1} \in[0,1]: \Pi\left(\theta_{1} \mid b_{2}\right)=\min \left\{\frac{F\left(\theta_{1}\right)}{F\left(\phi_{1}\left(b_{2}\right)\right)}, 1\right\},
$$

\footnotetext{
${ }^{6}$ The feature that the private-value bidder (bidder 1) uses a strictly increasing bid function while an additional bidder with no private information (bidder 2) randomizes her bid already appears in Vickrey (1961, Appendix III). He constructs an equilibrium for first-price auctions without resale in a 2-bidder environment where one bidder has a private uniformly distributed valuation and the other has a commonly known strictly positive valuation. The structure of Vickrey's equilibrium differs from ours in important respects. First, the winning probability of Vickrey's commonly-known-value bidder tends to 0 as her valuation tends to 0 , while the speculator wins with a positive probability although her use value equals 0. Second, Vickrey's commonly-known-value bidder obtains a strictly positive equilibrium payoff while the speculator obtains 0 . Third, in Vickrey's equilibrium the private-value bidder wins the auction with probability 0 if her valuation is sufficiently close to 0 , while in our equilibrium every non-zero type wins with positive probability.
} 
where we define $\phi_{1}\left(b_{2}\right)=1$ for all $b_{2}>\beta_{1}(1)$.

$$
\forall b_{2} \geq 0: T\left(b_{2}\right) \in \arg \max _{p \geq 0} p\left(F\left(\phi_{1}\left(b_{2}\right)\right)-F(p)\right)
$$

Several properties of the equilibrium are worth mentioning. First, the equilibrium is

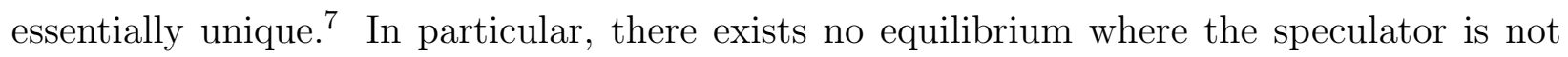
active. Second, although the speculator plays an active role, speculation is not profitable: bidder 2's payoff equals 0 because bidder 1's bid function (7) is such that, for all bids $b$ in the range of bidder 1's bid function, bidder 2's discounted resale profit equals $b$ if she bids $b$. Third, the final allocation is inefficient with positive probability, even in the limit $\delta \rightarrow 1 .^{8}$ The reason is that, because the losing bid remains private, bidder 1 retains some private information when she enters the resale market, so that with positive probability the resale offer is unacceptably high. ${ }^{9}$ Because in the absence of a resale opportunity the allocation would be efficient, we obtain the conclusion that a resale opportunity can be detrimental to efficiency.

The proof of Proposition 1 can be summarized as follows. The core step is to show that in any perfect Bayesian equilibrium bidder 2's payoff equals 0. Bidder 2's payoff is, for each possible bid, determined by the set of bidder-1 types against which the bid wins. Hence, the 0-payoff condition for bidder 2 determines a unique strictly increasing bid function for

\footnotetext{
${ }^{7}$ If condition (10) is satisfied for two functions $T=T^{\prime}$ and $T=T^{\prime \prime}$ then $\operatorname{Pr}\left[T^{\prime}\left(\tilde{b}_{2}\right)=T^{\prime \prime}\left(\tilde{b}_{2}\right)\right]=1$, where $\tilde{b}_{2}$ denotes a random variable with distribution $H$. This follows because Edlin and Shannon's (1998) strict monotone comparative statics techniques show that the arg max-correspondence from which $T$ is selected in (10) is strictly increasing and thus its set of points of multiple-valuedness is countable (and thus has measure 0 according to $H$ ).

${ }^{8}$ An inefficiency would also occur in an model without resale if bidder 2 had a commonly known strictly positive use value, but the equilibrium outcome would still be qualitatively different from that of Proposition 1 ; cf. footnote 6 .

${ }^{9}$ This is not saying that the final allocation would be efficient if the losing bid were revealed. Krishna (2002, Section 4.4) considers first-price auctions with two asymmetric private-value bidders where the loser's bid is revealed and the winner can make a take-it-or-leave-it offer to the loser. Krishna shows that the anticipation of resale prevents bidders from using strictly increasing bid functions. Thus, bidders retain some private information when they enter the resale market.
} 
bidder 1. Finally, there exists a unique bid distribution for bidder 2 such that no bidder-1 type wants to deviate from her bid.

Lemma 1 contains the main insight towards establishing the 0 -profit claim for bidder 2 . The lemma shows that bidder 2 makes, with positive probability, arbitrarily small bids. If bidder 2's bid distribution has an atom at the infimum bid $\underline{b}$, the proof is as follows. Suppose that $\underline{b}>0$. Because no bidder- 1 type bids higher than her use value, the bid $\underline{b}>0$ wins with positive probability. Hence, to avoid expected losses for bidder 2 the resale price $T(\underline{b})$ must exceed $\underline{b} / \delta$, and there must exist some bidder-1 types greater than $T(\underline{b})$ who are overbid by bidder 2's bid $\underline{b}$ in the auction and accept the resale price $T(\underline{b})$. For these bidder-1 types it is profitable to deviate to a bid slightly above $\underline{b}$. In doing so, they win against bidder 2's bid $\underline{b}$ and avoid the higher discounted resale price $\delta T(\underline{b})$ - a contradiction. If bidder 2's bid distribution has no atom at $\underline{b}$ the proof is similar, but some limit arguments are needed; the details can be found in the proof below.

Lemma 1 A tuple $\left(\beta_{1}, H, T, \Pi\right)$ is a perfect Bayesian equilibrium for the first-price auction with resale only if $H(b)>0$ for all $b>0$.

Proof. As a preliminary step, observe that a bidder-1 type who wins with positive probability will not bid above her use value because she cannot resell the good at any positive price,

$$
\forall \theta_{1} \in[0,1], H\left(\beta_{1}\left(\theta_{1}\right)\right)>0: \beta_{1}\left(\theta_{1}\right) \leq \theta_{1} .
$$

Let $\underline{b}=\inf \{b \mid H(b)>0\}$. Suppose that $\underline{b}>0$. Let $U_{2} \geq 0$ denote bidder 2's equilibrium payoff.

Suppose that $H(\underline{b})=0$; i.e., no atom at $\underline{b}$ (the case $H(\underline{b})>0$ is treated in the text above). Let $\Theta=\left\{\theta_{1} \mid \beta_{1}\left(\theta_{1}\right) \leq \underline{b}\right\}$. By (11),

$$
[0, \underline{b}] \subseteq \Theta
$$

For all $b>\underline{b}$ with $u_{2}(b)=U_{2}$,

$$
0 \leq u_{2}(b)=\operatorname{Pr}\left[\beta_{1}\left(\tilde{\theta}_{1}\right)<b\right]\left(-b+\operatorname{Pr}\left[\tilde{\theta}_{1} \geq T(b) \mid \beta_{1}\left(\tilde{\theta}_{1}\right)<b\right] \delta T(b)\right) .
$$


By $(12), \operatorname{Pr}\left[\beta_{1}\left(\tilde{\theta}_{1}\right)<b\right]>0$, hence

$$
\forall b>\underline{b}, u_{2}(b)=U_{2}: \operatorname{Pr}\left[\tilde{\theta}_{1} \geq T(b) \mid \beta_{1}\left(\tilde{\theta}_{1}\right)<b\right] \delta T(b)>\underline{b} .
$$

Therefore, $T(b)>\underline{b}$ and thus

$$
\begin{aligned}
\operatorname{Pr}\left[\tilde{\theta}_{1}<T(b) \mid \beta_{1}\left(\tilde{\theta}_{1}\right)<b\right] & \underset{(12)}{\geq} \operatorname{Pr}\left[\tilde{\theta}_{1}<\underline{b} \mid \beta_{1}\left(\tilde{\theta}_{1}\right)<b\right] \\
& \operatorname{Pr}\left[\tilde{\theta}_{1}<\underline{b}\right]=F(\underline{b}) .
\end{aligned}
$$

Defining $\xi=1-F(\underline{b})<1$ and using (13), we find

$$
\forall b>\underline{b}, u_{2}(b)=U_{2}: \xi \delta T(b)>\underline{b} .
$$

Let $\left(b^{m}\right)_{m \in \mathbb{N}}$ be any sequence such that $b^{m} \rightarrow \underline{b}$ as $m \rightarrow \infty$, and $u_{2}\left(b^{m}\right)=U_{2}$ and $b^{m}>\underline{b}$ for all $m$. Because some type $\theta^{m}$ accepts the resale price $T\left(b^{m}\right)$, there exists a sequence $\left(\theta^{m}\right)_{m \in \mathbb{N}}$ such that $\beta_{1}\left(\theta^{m}\right)<b^{m}$ and $\theta^{m} \geq T\left(b^{m}\right)$ for all $m$.

Fix a bid $b_{1} \in(\underline{b}, \underline{b} / \xi)$. We now show that a deviation to bid $b_{1}$ is profitable for type $\theta^{m}$ if $m$ is sufficiently large. Let $m$ be so large that $\beta_{1}\left(\theta^{m}\right)<b_{1}$. Then

$$
\begin{aligned}
& u_{1}\left(b_{1}, \theta^{m}\right)-u_{1}\left(\beta_{1}\left(\theta^{m}\right), \theta^{m}\right) \\
& =H\left(b_{1}\right)\left(\theta^{m}-b_{1}\right)-H\left(\beta_{1}\left(\theta^{m}\right)\right)\left(\theta_{1}-\beta_{1}\left(\theta^{m}\right)\right) \\
& -\int_{\left(\beta_{1}\left(\theta^{m}\right), b_{1}\right]} \mathbf{1}_{\theta^{m} \geq T(b)} \delta\left(\theta^{m}-T(b)\right) \mathrm{d} H(b) \\
& \stackrel{(14)}{\geq} H\left(b_{1}\right)\left(\theta^{m}-b_{1}\right)-H\left(\beta_{1}\left(\theta^{m}\right)\right)\left(\theta_{1}-\beta_{1}\left(\theta^{m}\right)\right) \\
& -\left(\delta \theta^{m}-\underline{b} / \xi\right)\left(H\left(b_{1}\right)-H\left(\beta_{1}\left(\theta^{m}\right)\right)\right) \\
& \geq\left(H\left(b_{1}\right)-H\left(\beta_{1}\left(\theta^{m}\right)\right)\left(\underline{b} / \xi-b_{1}\right)-H\left(\beta\left(\theta^{m}\right)\right)\left(\theta_{1}-\beta_{1}\left(\theta^{m}\right)\right)\right. \text {. }
\end{aligned}
$$

This implies $u_{1}\left(b_{1}, \theta^{m}\right)>u_{1}\left(\beta_{1}\left(\theta^{m}\right), \theta^{m}\right)$ for large $m$ because $H\left(\beta_{1}\left(\theta^{m}\right)\right) \rightarrow 0$ as $m \rightarrow \infty$. This contradicts (5).

$Q E D$

We now provide the proof of Proposition 1. 
Proof of Proposition 1. We begin with the "only-if"-part. The first step is to show that bidder 2's payoff $U_{2}=0$ in any perfect Bayesian equilibrium. Suppose to the contrary that $U_{2}>0$. By Lemma 1, arbitrarily small bids yield the positive payoff $U_{2}$. Hence, a positive mass of bidder- 1 types must be bidding 0 . But an argument similar to that in the proof of Lemma 1 shows that for some of these types it is profitable to deviate to a small positive bid-a contradiction.

Bidder 1's bid function $\beta_{1}$ is strictly increasing and continuous (so that it has a continuous inverse $\left.\phi_{1}\right),{ }^{10}$ and every positive type bids lower than her use value (because she cannot sell the good at any positive price). Hence, bidder 2's post-auction beliefs $\Pi$ satisfy (9) and her resale price function $T$ satisfies (10). Therefore, bidder 2's payoff function (2) is given by

$$
\forall b_{2} \geq 0: u_{2}\left(b_{2}\right)=F\left(\phi_{1}\left(b_{2}\right)\right)\left(-b_{2}+\delta \max _{p \geq 0} \frac{F\left(\phi_{1}\left(b_{2}\right)\right)-F(p)}{F\left(\phi_{1}\left(b_{2}\right)\right)} p\right) .
$$

Bidder 2's bid distribution $H$ has the support $\left[0, \beta_{1}(1)\right] .{ }^{11}$ By Berge's Maximum Theorem, $u_{2}$ is continuous. Hence, any bid in the support of $H$ yields the equilibrium payoff:

$$
\forall b_{2} \in\left[0, \beta_{1}(1)\right]: u_{2}\left(b_{2}\right)=U_{2}=0 .
$$

Formula (7) is straightforward from (15) and (16).

Finally, $H$ is determined by the condition that the bid function $\beta_{1}$ is locally optimal. For any $b \in\left(0, \beta_{1}(1)\right)$, consider the effect of a marginal bid increase of type $\theta_{1}=\phi_{1}(b)$. With marginal probability $H^{\prime}(b)$, she gains the auction winning payoff $\theta_{1}-b$ and loses the resale payoff $\theta_{1}-T(b)$, and with her winning probability $H(b)$ there is a marginal increase of her auction payment. Therefore, her first-order condition is

$$
0=\left.\frac{\partial u_{1}}{\partial b}\left(b, \theta_{1}\right)\right|_{\theta_{1}=\phi_{1}(b)}=H^{\prime}(b)\left(\phi_{1}(b)-b-\delta\left(\phi_{1}(b)-T(b)\right)-H(b) .\right.
$$

\footnotetext{
${ }^{10}$ All this follows from standard arguments. Using $\delta<1$ one shows that bidder 1's payoff function (1) has strictly increasing differences, hence $\beta_{1}$ is weakly increasing. If $\beta_{1}$ had a flat part, optimality of bidder 2's bid distribution (6) would prevent her from making bids just below the flat part, which contradicts the optimality of $\beta_{1}$. If $\beta_{1}$ had a jump, bidder 2 would also not bid in the resulting gap of bidder 1's bid distribution, which contradicts the optimal bidding condition (5) for types just above the jumping type.

${ }^{11}$ The upper end of the support is $\beta_{1}(1)$ because no bidder will bid higher than necessary to win for sure. The lower end is 0 by Lemma 1 . If the distribution had a gap, some bidder- 1 type would be bidding unnecessarily high.
} 
This yields a differential equation for $H$ which together with the boundary condition $H\left(\beta_{1}(1)\right)=$ 1 has the unique solution (8). The above argument is incomplete because one cannot assume that $H$ is differentiable. However, revealed preference arguments for bidder 1 can be used to show that $H$ is Lipschitz continuous on $\left[b^{\prime}, \beta_{1}(1)\right]$ for all $b^{\prime}>0$, so that $H$ is differentiable almost everywhere and the above arguments go through.

The main step in the proof of the "if"-part of Proposition 1 is that bidder 1's payoff function (1) is quasi-concave so that bidder 1's bid function (7) is globally optimal; the proof is straightforward.

$Q E D$

REMARK 1: If the initial seller sets a small reserve price, the speculator still plays an active role. ${ }^{12}$ Precisely, there is no equilibrium where the speculator does not play an active role. To see this, consider a first-price auction with a reserve price $r>0$ and suppose that bidder 2 wins the auction with probability 0 . This implies that she does not submit a bid of $r$ or greater because such a bid would win against bidder- 1 types below $r$. Hence, no bidder- 1 type will bid more than $r$. But then it is profitable for bidder 2 to deviate to a bid just above $r$ and win the auction, provided $r$ is not too large. Hence, we have a contradiction.

REMARK 2: The speculator still plays an active role if we extend the bidders' preferences by assuming a small bid preparation cost (to be paid simultaneously with submitting any strictly positive bid). The argument is the same as with a small reserve price.

REMARK 3: The equilibrium remains valid with free entry of multiple speculators: abstaining is optimal for a second speculator because she obtains the same zero payoff as bidder 2 from any bid in $\left[0, \beta_{1}(1)\right]$. There also exist equilibria with multiple active speculators: bidder 1 still uses the bid function (7), and the distribution function for the maximum bid among all speculators (including bidder 2) is given by (8).

\footnotetext{
${ }^{12} \mathrm{~A}$ reserve price that is revenue maximizing in the absence of resale will shut out the speculator (because this price exceeds the resale profit even if the speculator wins the initial auction for sure).
} 
REMARK 4: The equilibrium construction of Proposition 1 can be extended to environments where bidder 2 has a known use value $\theta_{2}>0$. Two properties of the equilibrium are worth emphasizing. First, the initial seller's revenue is higher than in the absence of a resale opportunity if $\delta$ is close to 1 . Second, bidder 2's payoff is strictly positive, but tends to 0 as $\delta \rightarrow 1$. To see the revenue claim, observe first that if a bidder- 1 type below $\delta \theta_{2}$ wins the auction then she will offer the good for resale to bidder 2 at price $\theta_{2}$. Hence, a Bertrand argument shows that $\delta \theta_{2}$ is a lower bound for the winning bid in the auction. In the absence of a resale opportunity (see Vickrey, 1961, Appendix III, for the construction of an equilibrium; cf. footnote 6 ) bidder 2's highest equilibrium bid $\bar{b}<\theta_{2}$. Hence an upper bound for the winning bid is given by $\bar{b}<\delta \theta_{2}$ if $\delta$ is close to 1 . To see the second property mentioned above, concerning bidder 2's equilibrium payoff, one first uses an argument similar to that of Lemma 1 to show that the lower end of bidder 2's bid distribution $\underline{b}<\theta_{2}$. Because with bid $\underline{b} \in\left[\delta \theta_{2}, \theta_{2}\right]$ bidder 2 does not win against the types above $\underline{b}$, bidder 2 's payoff cannot exceed $F(\underline{b})\left(\theta_{2}-\underline{b}\right)$. On the other hand, the expression $F(b)\left(\theta_{2}-b\right)$ is a lower bound for bidder 2's payoff with any bid $b \in\left[\delta \theta_{2}, \theta_{2}\right]$ because the bidder-1 types above $\delta \theta_{2}$ will not bid higher than their use values. Hence, bidder 2's payoff equals $\max _{b \in\left[\delta \theta_{2}, \theta_{2}\right]} F(b)\left(\theta_{2}-b\right)$, which is positive and tends to 0 as $\delta \rightarrow 1$.

\section{Second-price/English auctions with resale}

In this section we construct and discuss a continuum of pure-strategy perfect Bayesian equilibria for second-price auctions with resale where the speculator plays an active role. Here, $\beta_{1}$ denotes bidder 1's bid as a function of her use value and $\hat{b}_{2}$ denotes bidder 2's bid (in contrast to the first-price auction setting, we do not consider randomized bidding of the speculator because it is not needed for the equilibrium construction). For any bidder-1 bid $b_{1} \geq 0$, the probability distribution $\Pi\left(\cdot \mid b_{1}\right)$ describes bidder 2's post-auction beliefs about bidder 1's use value if bidder 2 wins at price $b_{1} . T\left(b_{1}\right)$ denotes bidder 2's resale offer. For all 
$b_{1} \geq 0$ and $\theta_{1} \in[0,1]$, bidder 1 's payoff is

$$
u_{1}\left(b_{1}, \theta_{1}\right)= \begin{cases}\theta_{1}-\hat{b}_{2} & \text { if } b_{1} \geq \hat{b}_{2}, \\ \delta\left(\theta_{1}-T\left(b_{1}\right)\right) & \text { if } b_{1}<\hat{b}_{2}, T\left(b_{1}\right) \leq \theta_{1}, \\ 0 & \text { if } b_{1}<\hat{b}_{2}, T\left(b_{1}\right)>\theta_{1} .\end{cases}
$$

For all bids $b_{2} \geq 0$, bidder 2 's payoff is

$$
u_{2}\left(b_{2}\right)=E\left[-\beta_{1}\left(\tilde{\theta}_{1}\right) \mathbf{1}_{b_{2}>\beta_{1}\left(\tilde{\theta}_{1}\right)}+\delta T\left(\beta_{1}\left(\tilde{\theta}_{1}\right)\right) \mathbf{1}_{b_{2}>\beta_{1}\left(\tilde{\theta}_{1}\right), \tilde{\theta}_{1} \geq T\left(\beta_{1}\left(\tilde{\theta}_{1}\right)\right)}\right]
$$

The perfect Bayesian equilibrium conditions are Bayes rule (19), optimality of the resale offer (20), and optimal bidding $(21,22)$, as shown in the following

Definition 2 A tuple $\left(\beta_{1}, \hat{b}_{2}, T, \Pi\right)$ is a perfect Bayesian equilibrium for the second-price auction with resale if

$$
\forall \theta_{1} \in[0,1], b_{1} \geq 0: \int_{\left[0, b_{1}\right]} \Pi\left(\theta_{1} \mid b\right) d G(b)=\operatorname{Pr}\left[\tilde{\theta}_{1} \leq \theta_{1}, \beta_{1}\left(\tilde{\theta}_{1}\right) \leq b_{1}\right]
$$

where $G$ denotes the distribution function for $\beta_{1}\left(\tilde{\theta}_{1}\right)$,

$$
\forall b_{1} \geq 0: T\left(b_{1}\right) \in \arg \max _{p \geq 0}\left(1-\Pi_{-}\left(p \mid b_{1}\right)\right) p
$$

where $\Pi_{-}\left(p \mid b_{1}\right)=\lim _{p^{\prime} / p} \Pi\left(p^{\prime} \mid b_{1}\right)$ denotes the posterior probability that bidder 1's use value is less than $p$,

$$
\forall \theta_{1} \in[0,1]: \beta_{1}\left(\theta_{1}\right) \in \arg \max _{b_{1} \geq 0} u_{1}\left(b_{1}, \theta_{1}\right)
$$

and

$$
\hat{b}_{2} \in \arg \max _{b_{2} \geq 0} u_{2}\left(b_{2}\right) .
$$

Proposition 2 describes the equilibria. Bidder 1's bid function (23) is such that all types below some fixed parameter $\theta^{*}$ pool at the bid 0 (or, equivalently, abstain from the auction) while the types above $\theta^{*}$ bid their use values. In particular, in equilibrium bidder 1 does not make a bid in $\left(0, \theta^{*}\right) .{ }^{13}$ Bidder 2 's bid (24) lies in $\left(0, \theta^{*}\right)$ and is such that the bidder-1 type

\footnotetext{
${ }^{13}$ The shape of bidder 1's bid function - a pool of low types, a gap in the bid distribution, and above that a strictly increasing segment - resembles the symmetric equilibrium bid functions derived in Jehiel and
} 
$\theta^{*}$ is indifferent between overbidding bidder 2 and waiting for a resale offer. Bidder 2's resale offer function (25) follows from her post-auction beliefs (26). If bidder 2 wins at price 0 she believes that bidder 1 's type is chosen from $\left[0, \theta^{*}\right]$; if bidder 2 wins at a price in $\left(\theta^{*}, 1\right]$ she believes that bidder 1's type equals the auction price; if bidder 2 wins at an off-equilibrium price in $\left(0, \theta^{*}\right)$ she believes that bidder 1 's type equals $\theta^{*}{ }^{14}$

Proposition 2 For every $\theta^{*} \in[0,1]$, the second-price auction with resale has a perfect Bayesian equilibrium $\left(\beta_{1}, \hat{b}_{2}, T, \Pi\right)$ with the properties (23), (24), (25), and (26).

$$
\begin{aligned}
\beta_{1}\left(\theta_{1}\right) & = \begin{cases}0 & \text { if } \theta_{1} \in\left[0, \theta^{*}\right), \\
\theta_{1} & \text { if } \theta_{1} \in\left(\theta^{*}, 1\right] .\end{cases} \\
\hat{b}_{2} & =\theta^{*}-\delta\left(\theta^{*}-T(0)\right) .
\end{aligned}
$$

For all $b_{1} \in[0,1]$,

$$
T\left(b_{1}\right)= \begin{cases}\in \arg \max _{p \geq 0} p\left(F\left(\theta^{*}\right)-F(p)\right) & \text { if } b_{1}=0, \\ \theta^{*} & \text { if } b_{1} \in\left(0, \theta^{*}\right), \\ b_{1} & \text { if } b_{1} \in\left(\theta^{*}, 1\right] .\end{cases}
$$

For all $\theta_{1} \in[0,1]$ and $b_{1} \in[0,1]$,

$$
\Pi\left(\theta_{1} \mid b_{1}\right)= \begin{cases}\min \left\{F\left(\theta_{1}\right) / F\left(\theta^{*}\right), 1\right\} & \text { if } b_{1}=0 \text { and } \theta^{*}>0, \\ \mathbf{1}_{\theta_{1} \geq \theta^{*}} & \text { if } b_{1} \in\left(0, \theta^{*}\right), \\ \mathbf{1}_{\theta_{1} \geq b_{1}} & \text { if } b_{1} \in\left(\theta^{*}, 1\right] .\end{cases}
$$

Moldovanu (2000) and Haile (2000) for second-price auctions with a binding reserve price in environments with positive externalities (such as the externalities arising from resale markets with complete information). In these models, the size of the pool is determined by a trade-off arising from the fact that the positive externality vanishes if the initial seller keeps the good. In particular, in contrast to our model, there is no pooling in the absence of a reserve price, and there is no continuum of possible equilibrium pool sizes.

${ }^{14}$ The equilibrium with $\theta^{*}=1$ is similar in spirit to an equilibrium constructed by Zheng (2000, Section 5.2) for an optimal auction a la Myerson (1981) with two private-value bidders. According to Zheng's construction, one bidder, if her use value is sufficiently high submits a high bid that wins for sure, and otherwise she abstains. The other bidder bids the reserve price if her use value is sufficiently high and otherwise she abstains. Zheng emphasizes that according to his equilibrium the resale opportunity reduces the initial seller's revenue. 
Several properties of the equilibria of Proposition 2 are worth emphasizing. First, equilibria where the speculator plays an active role $\left(\theta^{*}>0\right)$ coexist with an equilibrium where both bidders bid their use values so that no active resale market arises $\left(\theta^{*}=0\right)$. Second, speculation is profitable in any equilibrium with $\theta^{*}>0$ because the speculator wins at price 0 and sells at a positive price. In fact, Proposition 2 shows that any speculator payoff between 0 and $\delta \max _{p \geq 0} p(1-F(p))$ is supported by an equilibrium. Third, the final allocation is inefficient with positive probability in any equilibrium with $\theta^{*}>0$, even in the limit $\delta \rightarrow 1$. The inefficiency arises because the losing bidder-1 types pool at the same bid, which implies that bidder 1 retains some private information when she enters the resale market. Forth, bidder 1's equilibrium strategy is not weakly dominated, and bidder 2's equilibrium strategy is not weakly dominated if $\delta$ is sufficiently close to 1 . In particular, if $\delta>\hat{b}_{2}$ then bidder 2's equilibrium strategy is not dominated by a strategy where she makes a bid $b_{2}^{\prime}<\hat{b}_{2}$ (bidder-1 types close to 1 may bid just below $\hat{b}_{2}$, in which case bidder 2 's profit conditional on winning at a price just below $\hat{b}_{2}$ approximates $\left.\delta-\hat{b}_{2}>0\right) .{ }^{15}$

The proof of Proposition 2 is straightforward. No bidder-1 type profits from a deviation to a bid in $\left(0, \hat{b}_{2}\right)$ because, by the off-equilibrium beliefs defined in (26), the deviation leads to the high resale price $\theta^{*}$ rather than the resale price $T(0)<\theta^{*}$ that results from bidding $0 .{ }^{16}$ By (24), all bidder-1 types below $\theta^{*}$ prefer bidding 0 and waiting for a resale offer to overbidding bidder 2 . The bidder- 1 types above $\theta^{*}$ have the opposite preference. For bidder 2 any bid in $\left(0, \theta^{*}\right]$ is equally good because she wins at price 0 ; it is harmful to bid higher

\footnotetext{
${ }^{15}$ The second-price auction without resale also has a continuum of equilibria parameterized by $\theta^{*}$ where bidder 1 uses a bid function $\beta_{1}$ satisfying (23) and bidder 2 submits the bid $\theta^{*}$, but the equilibria with $\theta^{*}>0$ are in weakly dominated strategies and they break down if an arbitrarily small reserve price is introduced (cf. Remark 5). See Blume and Heidhues $(2001,2004)$ for a characterization of the entire set of equilibria of the second-price auction without resale in environments with independent private values having identical support, and for a proof that only the bid-your-value equilibrium remains if an arbitrarily small reserve price is introduced.

${ }^{16}$ The off-path beliefs defined in (26) satisfy the intuitive criterion. There exist beliefs different from (26) that also support our equilibria. For example, the off-equilibrium beliefs could be identical to bidder 2's beliefs when she wins at price 0 (this was suggested to us by Bill Zame). More generally, our equilibria are supported by any off-equilibrium beliefs that lead to a resale price greater than or equal to $T(0)$.
} 
than $\theta^{*}$ because in the event that bidder 2 needs such a high bid in order to win, she must sell at a price equal to her auction payment.

REMARK 5: If the initial seller sets a reserve price that is not too large, equilibria with sufficiently large parameters $\theta^{*}$ remain valid. ${ }^{17}$ More precisely, with reserve price $r$ the equilibria such that $\max _{p \geq 0} p\left(F\left(\theta^{*}\right)-F(p)\right) \geq r F\left(\theta^{*}\right)$ remain valid.

REMARK 6: The equilibria remain essentially valid if we extend the bidders' preferences by assuming a small bid preparation cost (to be paid when submitting a strictly positive bid). In fact, such costs would add additional stability to our equilibria because the bidder-1 types below the resale price $T(0)$, who in the absence of bid preparation costs are indifferent between bidding 0 and bidding, say, their values, would find it strictly optimal to bid 0 .

REMARK 7: The equilibria remain valid with free entry of multiple speculators. A second speculator can conceivably make a profit only if she overbids bidder 2's bid $\hat{b}_{2}$. Her best hope is a bid in the interval $\left(\hat{b}_{2}, \theta^{*}\right.$ (if she needs a bid above $\theta^{*}$ in order to win, the highest use value in the market does not exceed her payment in the auction). Any such bid yields a resale revenue smaller than $T(0)$, and hence smaller than $\hat{b}_{2}$, showing that she would make a loss.

REMARK 8: The equilibrium construction of Proposition 2 can be extended to environments where bidder 2 has a known use value $\theta_{2}>0$. For every $\theta^{*}>\theta_{2}$ and $\delta$ sufficiently close to 1 , there exists like before an equilibrium where all bidder- 1 types above $\theta^{*}$ bid their use values and all types below $\theta^{*}$ submit losing bids. The reason this equilibrium construction breaks down for small discount factors $\delta$ is that it would optimal for bidder 2 to consume the good upon winning.

\footnotetext{
${ }^{17}$ If a reserve price that is revenue maximizing in the absence of resale is set, all equilibria with $\theta^{*}>0$ break down. The argument is similar to the first-price auction setting, cf. footnote 12 .
} 
REMARK 9: Final allocation and initial seller revenue differ across standard auction formats. ${ }^{18}$ Precisely, none of the equilibria of Proposition 2 for second-price and English auctions with resale yields the same final allocation (and only one yields the same revenue) as the equilibrium of Proposition 1 for a first-price or Dutch auction with resale. According to the equilibrium of Proposition 1, every non-zero bidder-1 type wins the initial auction with positive probability. But in every $\theta^{*}$-equilibrium with $\theta^{*}>0$, bidder 2 's resale price is positive, so that any sufficiently small bidder- 1 type obtains the good with probability 0 , and if $\theta^{*}=0$ the allocation is efficient.

\section{Extension to multiple private-value bidders}

This section summarizes the extension of our model to environments with multiple symmetric independent private value bidders (henceforth called regular bidders) and a speculator. Details can be found in an online supplement. The main additional assumptions are that the value distribution $F$ for the regular bidders has an increasing hazard rate, and that we focus on equilibria such that all regular bidders use the same bid function that is strictly increasing in the winning range and such that all bidders who expect to win with probability 0 bid 0 . Concerning resale mechanisms we cover two possibilities: the resale seller may use any standard auction with an optimal reserve price, or she may be a mechanism designer who uses an optimal mechanism.

The basic result that a speculator can play an active role and cause a distortion of the final allocation in any standard auction remains valid, although in the case of first-price and Dutch auctions this will depend on the number of bidders, the value distribution, and the discount factor. The result that a speculator can make profits in second-price and English auctions, but not in a first-price or Dutch auction, also remains true.

\footnotetext{
${ }^{18}$ The failure of allocation equivalence contrasts earlier comparisons of standard auction formats with resale such as Haile (2003), where the focus is mainly on revenue comparisons because appropriate assumptions about the environment and the equilibria usually guarantee that the final allocation is efficient and hence identical across auction formats.
} 
It is particularly instructive to understand why the speculator can play an active role in a first-price or Dutch auction with any number $n \geq 2$ of regular bidders. ${ }^{19}$ Consider a distribution $F$ such that, except at points close to 1, the density is that of an exponential distribution; i.e., $F(\theta)=1-e^{-z \theta}$, for some $z \in(0,1)$. When the point 1 is approached, the density suddenly peaks. Such a distribution has a constant hazard rate up to values close to 1 , but then the hazard rate increases sharply. Suppose that the speculator does not play an active role. Then all regular bidders use the standard no-resale equilibrium bid function $\beta^{\mathrm{I}}$. To obtain a contradiction we show that if the discount factor $\delta$ is sufficiently close to 1 , a profitable deviation for the speculator is to bid $\beta^{\mathrm{I}}(1)$ in the auction and offer the good at a price just below 1 in the resale market. The argument is as follows. By definition of $\beta^{\mathrm{I}}$, the bid $\beta^{\mathrm{I}}(1)$ equals the expectation of the distribution $F^{n-1}$, which approximates

$$
\eta(z, n)=(n-1) \frac{1}{z} \int_{0}^{z} \tau e^{-\tau}\left(1-e^{-\tau}\right)^{n-2} \mathrm{~d} \tau+1-\left(1-e^{-z}\right)^{n-1} .
$$

The resale revenue approximates the probability that at least one bidder has a use value close to 1 ,

$$
\rho(z, n)=1-\left(1-e^{-z}\right)^{n} .
$$

Using the Taylor expansion for $e^{-z}$, one finds that for $z \approx 0, \rho(z, n)=1-z^{n}+o\left(z^{n}\right)$ and $\eta(z, n)=1-\frac{1}{n} z^{n-1}+o\left(z^{n-1}\right) .{ }^{20}$ Therefore, $\rho(z, n)>\eta(z, n)$ for small $z$. Hence, when the discount factor is close to 1 , the bid $\beta^{\mathrm{I}}(1)$ is profitable for the speculator, a contradiction to the claim that the speculator does not play an active role.

A question that arises specifically in environments with multiple regular bidders is whether the presence of a resale opportunity increases or decreases initial seller revenue (the answer is trivial in environments with a single regular bidder because in the absence of resale the revenue is zero). Concerning first-price and Dutch auctions, the answer is that resale never reduces initial seller revenue, and strictly increases it if the speculator plays an active role. In a second-price or English auction a resale opportunity may strictly increase or decrease

\footnotetext{
${ }^{19}$ It is easy to find examples where the speculator does not play an active role. The uniform distribution $F$ together with any discount factor $\delta$ and any $n \geq 2$ will do.

${ }^{20}$ We use the notation $\mathcal{o}\left(z^{l}\right)$ for any function $\epsilon(z)$ with the property $\epsilon(z) / z^{l} \rightarrow 0$ as $z \rightarrow 0$.
} 
initial seller revenue: equilibria with a low winning probability of the speculator correspond to a revenue increase, while equilibria with a high winning probability of the speculator correspond to a revenue decrease.

A final result is that the presence of a resale opportunity can decrease initial seller revenue in second-price and English auctions in an extended game where the initial seller can set an arbitrary reserve price for the initial auction. This result is important because it shows that the impact of a resale opportunity on initial seller revenue does not always vanish in environments where both the initial seller and the resale seller have access to the same class of sales mechanisms (second-price or English auctions with arbitrary reserve prices).

Department of Economics, University of California at Santa Barbara, CA 93106-9210, U.S.A.,garratt@econ.ucsb.edu

Department of Economics, University of Bonn, Adenauerallee 24-26, 53113 Bonn, Germany, ttroeger@uni-bonn.de

\section{References}

Ausubel, L., And P. Cramton (1999), "The Optimality Of Being Efficient," mimeo, University of Maryland.

Bikchandani, S., And C. Huang (1989) "Auctions With Resale Market: An Exploratory Model of Treasury Bill Markets," Review of Economic Studies 2, 311-340.

Blume, A., And P. Heidues (2001) "All Equilibria of the Vickrey Auction," University of Pittsburg and Social Science Research Center (WZB) Working Paper, available at www.wzberlin.de.

- (2004) "All Equilibria of the Vickrey Auction," Journal of Economic Theory 114, 170-177.

Bose, S., And G. Deltas (2002) "Welcoming the Middlemen: Restricting Competition in Auctions by Excluding Consumers," in Current Trends in Economics: Theory and Applications (Studies in Economic Theory, vol. 8), ed. by Aliprantis, Alkan, and Yannelis, Springer-Verlag, 119-131. 
Calzolari, G., and A. Pavan (2002), "Monopoly With Resale," mimeo, University of Toulouse.

Edlin, A., And C. Shannon (1998), "Strict Monotonicity In Comparative Statics," Journal of Economic Theory 81, 201-219.

Garratt, R., And T. Tröger (2003), "Speculation in Second-Price Auctions with Resale," http://econpapers.hhs.se/paper/wpawuwpga/0305003.htm.

Gupta, M., And B. Lebrun (1999), "First-Price Auctions With Resale," Economics Letters 64, 181-185.

Haile, P. (1999), "Auctions With Resale," mimeo, University of Wisconsin, Madison.

- (2000), "Partial Pooling At The Reserve Price In Auctions With Resale Opportunities," Games and Economic Behavior 33, 231-248.

- (2003), "Auctions with Private Uncertainty And Resale Opportunities," Journal of Economic Theory 108, 72-100.

Jehiel, P., And B. Moldovanu (1999), "Resale Markets And The Assignment of Property Rights," Review of Economic Studies 66, 971-999.

- (2000), "Auctions With Downstream Interaction Among Buyers," RAND Journal of Economics 31, 768-791.

Klemperer, P. (1999), "Auction Theory: A Guide to the Literature," Journal of Economic Surveys 13, 227-286.

Krishna, Vijay (2002), Auction Theory, Academic Press.

Milgrom, P. R. (1987), "Auction Theory," in Advances in Economic Theory, Fifth World Congress, ed. by T.F. Bewley, Cambridge University Press, Cambridge, U.K.

Myerson, R. (1981), "Optimal Auction Design," Mathematics of Operations Research 6, $58-73$.

Tröger, T. (2003), "Speculation in First-Price Auctions With Resale," http://econpapers.hhs.se/paper/wpawuwpmi/0308001.htm.

Vickrey, W. (1961), "Counterspeculation, Auctions, and Competitive Sealed Tenders," Journal of Finance 16, 8-37.

Zheng, C. (2000), "An Optimal Auction When Resale Cannot Be Prohibited," Northwest- 
ern University, CMS-EMS Discussion Paper 1303.

- (2002), "Optimal Auction With Resale," Econometrica 70, 2197-2224. 


\title{
Supplement to
}

\section{"Speculation in Standard Auctions with Resale"}

\author{
By Rod Garratt and Thomas Tröger
}

May 4, 2005

\begin{abstract}
We extend the model presented in the Note "Speculation in Standard Auctions with Resale" by the same authors to environments with multiple symmetric independentprivate-value bidders and prove the results stated in Section 5 of the Note.
\end{abstract}

KEYWORDS: standard auctions, speculation, resale, efficiency

JEL CODE: D44

In Section 1, we extend the model from Garratt and Tröger (2005), subsequently referred to as the Note, to environments with multiple private-value bidders. Section 2 deals with first-price and Dutch auctions. Section 3 is devoted to second-price auctions. Section 4 explains that the second-price auction results remain valid for English auctions. 


\section{Extending the model to multiple private-value bid- ders}

We consider environments with $n \geq 2$ risk-neutral bidders $i=1, \ldots, n$, called regular bidders, who are interested in consuming a single indivisible private good, and an additional riskneutral bidder $s$ called the speculator. Let $I=\{1, \ldots, n\}$. Let $\tilde{\theta}_{i} \in[0,1]$ denote the use value of bidder $i \in I$ for the good, and let $\tilde{\theta}_{s}=\theta_{s}=0$ denote the speculator's use value for the good. For all $i \in I \cup\{s\}$, let $\tilde{\theta}_{-i}^{(1)}=\max _{j \in I \backslash\{i\}} \tilde{\theta}_{j}$ denote the random variable

for the highest use value among bidders other than $i$. The random variables $\tilde{\theta}_{1}, \ldots, \tilde{\theta}_{n}$ are stochastically independent, and each $\tilde{\theta}_{i}(i \in I)$ is distributed according to the same distribution $F$. We assume that $F$ has a density $f$ that is positive and continuous on $[0,1]$ and identically 0 elsewhere. Because each bidder privately learns her realized use value before the interaction begins we say, following Vickrey (1961), that the bidders $1, \ldots, n$ are symmetric with independent private values (SIPV). We make the standard assumption that $F$ has a weakly increasing hazard rate.

Assumption 1 The mapping $\theta \mapsto f(\theta) /(1-F(\theta))$ is weakly increasing on $[0,1)$.

We consider a 2-period interaction. In period 1, the good is offered via a sealed-bid firstprice auction or second-price auction without reserve price (our analysis extends to English and Dutch auctions, see below). The highest bidder becomes the new owner of the good. Our results do not depend on the tieing rule, but to simplify some proofs we assume that the speculator loses all ties. The bidder who wins in period 1 either consumes the good in period 1 or offers the good for resale in period 2; if she fails to resell the good she consumes it in period 2. Period-2 payoffs are discounted according to a common factor $\delta \in(0,1)$.

\section{Modelling Period 1-the auction}

Because our focus is on the impact of the speculator, and the regular bidders are ex ante identical, we will focus on equilibria such that all regular bidders use the same bid function 
$\beta$ in period 1. This bid function is assumed to be strictly increasing in the winning range (this simplifies the computation of post-auction beliefs). Moreover, any regular-bidder type who does not expect to ever win the auction does not participate (formally, we assume such a type bids 0 , but we will show that with bid 0 she never wins). ${ }^{1}$

ASSUMPtion 2 In equilibrium, all regular bidders use the same bid function $\beta$ in period 1. For all $\theta, \theta^{\prime} \in[0,1]$ with $\theta>\theta^{\prime}$, we have $\beta(\theta)>\beta\left(\theta^{\prime}\right)$ if the bid $\beta\left(\theta^{\prime}\right)$ wins in equilibrium with positive probability, and have $\beta\left(\theta^{\prime}\right)=0$ otherwise.

For later use, let us introduce some notation for random vectors of bids and highest order statistics. For all $i \in I$, let $\tilde{b}_{i}=\beta\left(\tilde{\theta}_{i}\right)$ denote the random variable for $i$ 's bid. Let $\tilde{b}_{s}$ denote an independent random variable for the speculator's, possibly randomized, bid. For all $i \in I \cup\{s\}$, let $\tilde{\mathbf{b}}_{-i}=\left(\tilde{b}_{j}\right)_{j \in(I \cup\{s\}) \backslash\{i\}}$ denote the random vector of bids by bidders other than $i$, and let $\tilde{b}_{-i}^{(1)}=\max _{j \in(I \cup\{s\}) \backslash\{i\}} \tilde{b}_{j}$ denote the random variable for the highest among these bids. For all $i, j \in I \cup\{s\}$, let $\tilde{\mathbf{b}}_{-i-j}=\left(\tilde{b}_{k}\right)_{k \in(I \cup\{s\}) \backslash\{i, j\}}$ denote the random vector of bids by bidders other than $i$ and $j$, and let $\tilde{b}_{-i-j}^{(1)}=\max _{k \in(I \cup\{s\}) \backslash\{i, j\}} \tilde{b}_{k}$ denote the random variable for the highest among these bids.

The next assumption extends the bid revelation assumptions from the Note.

Assumption 3 After a first-price auction the winner's bid becomes public; the losers' bids remain private.

After a second-price auction, the losers' bids become public; the winner's bid remains private.

These bid revelation assumptions make the first-price auction setting strategically equivalent to the Dutch auction setting. Concerning the second-price auction, the bid revelation assumptions are such that after the auction the same information is revealed as after an

\footnotetext{
${ }^{1}$ As for further regularity properties of the bid function $\beta$, note that we will construct equilibria in the second-price auction case where $\beta$ is not continuous. In the first-price auction case, there will be a unique equilibrium, where $\beta$ is always continuous, but not necessarily differentiable.
} 
English auction. In Section 4 we will show that our second-price auction results remain valid in the English auction setting. ${ }^{2}$

By Assumption 3, all bidders make the same bid observations. It is thus reasonable to assume that any two bidders have identical post-auction beliefs about a third bidder even if an unexpected event occurs so that Bayesian updating is not possible, and to assume that it is commonly believed that all bidders have these beliefs. Moreover, it is reasonable to maintain stochastic independence of the post-auction beliefs across bidders because use values are stochastically independent ex ante. Hence, given any auction outcome, we can assume that post-auction beliefs are summarized by a vector of distributions $\Pi=\left(\Pi_{j}\right)_{j \in I}$, where $\Pi_{j}$ represents the post-auction belief about bidder $j$ 's use value. Note that $\Pi$ includes the beliefs about the resale seller's use value unless the resale seller is the speculator.

The post-auction beliefs that occur in our equilibrium constructions are represented by distributions of the following kind. For any $\underline{\theta}, \bar{\theta} \in[0,1]$ such that $\underline{\theta} \leq \bar{\theta}$, consider a bidder who infers from the observed bidding behavior in period 1 that bidder $i$ 's $(i \in I)$ use value belongs to the interval $[\underline{\theta}, \bar{\theta}]$. According to Bayes rule, the resulting posterior distribution function $\hat{F}_{[\underline{\theta}, \bar{\theta}]}$ for bidder $i$ 's use value is given by

$$
\hat{F}_{[\underline{\theta}, \bar{\theta}]}\left(\theta_{i}\right)= \begin{cases}\frac{F\left(\theta_{i}\right)-F(\underline{\theta})}{F(\bar{\theta})-F(\underline{\theta})} & \text { if } \theta_{i} \in[\underline{\theta}, \bar{\theta}), \\ 1 & \text { if } \theta_{i} \geq \bar{\theta}, \\ 0 & \text { if } \theta_{i}<\underline{\theta} .\end{cases}
$$

Note that $\hat{F}_{[\underline{\theta}, \bar{\theta}]}$ is a point distribution if $\bar{\theta}=\underline{\theta}$. If $\bar{\theta}>\underline{\theta}$, the distribution $\hat{F}_{[\underline{\theta}, \bar{\theta}]}$ has (on its support $[\underline{\theta}, \bar{\theta}])$ the same regularity properties as $F$ : a positive and continuous density and an increasing hazard rate. By Assumption 2, the case $\bar{\theta}>\underline{\theta}$ becomes relevant only if an off-equilibrium bid or bid 0 is observed.

\section{Modelling period 2 - the resale market}

\footnotetext{
${ }^{2}$ In the second-price auction setting, we expect that our equilibria remain valid even if the initial seller does not announce any information about the bids. This is because in the equilibria that we will construct, the only dependence of the outcome of the resale market on the auction bids is via the highest loser bid, and in a second-price auction the auction winner learns the highest loser bid from the price she pays.
} 
The period-2 environment where resale takes place is determined by the identity $i \in$ $I \cup\{s\}$ and use value $\theta_{i}$ of the resale seller (i.e., the auction winner) and by the postauction beliefs $\boldsymbol{\Pi}=\left(\Pi_{j}\right)_{j \in I}$. Any tuple $\left(\boldsymbol{\Pi}, \theta_{i}, i\right)$ is called a resale environment. A resale mechanism is a game form to be played in period 2 with players $I \cup\{s\}$, where an outcome is a probability distribution over who gets the good and a vector of side payments among the players, together with an equilibrium such that the participation constraints are satisfied; if multiple equilibria exist, we assume an equilibrium that is best for the resale seller is played. A resale mechanism is called optimal if there exists no other resale mechanism that yields a higher expected payoff for the resale seller. For any resale environment $\left(\boldsymbol{\Pi}, \theta_{i}, i\right)$, let $\hat{\mathcal{M}}\left(\boldsymbol{\Pi}, \theta_{i}, i\right)$ denote the resale mechanism used.

Assumption 4 For all resale environments $\left(\boldsymbol{\Pi}, \theta_{i}, i\right)$ where every standard auction with an optimal reserve price is an optimal resale mechanism, the resale mechanism $\hat{\mathcal{M}}\left(\boldsymbol{\Pi}, \theta_{i}, i\right)$ is a standard auction with an optimal reserve price.

Observe that, according to this assumption, in any resale environment where not all four standard auctions are optimal, any resale mechanism may be used. Hence, Assumption 4 covers two important special cases: the resale seller may always use any standard auction with an optimal reserve price, ${ }^{3}$ or she may always use an optimal resale mechanism. ${ }^{4}$ The first case is attractive because the cost of implementing a standard auction is negligible in many applications. The second case is attractive because it allows the resale seller to be

\footnotetext{
${ }^{3}$ Strictly speaking, an optimal reserve price may not always exist (because post-auction beliefs may be represented by non-smooth distributions). In such a resale environment, Assumption 4 allows any resale mechanism to be used. In particular, a standard auction with any reserve price that is arbitrarily close to being optimal may be used.

${ }^{4}$ If an optimal resale mechanism does not exist the resale seller may use any resale mechanism that is arbitrarily close to being optimal. Observe that, due to independent private values in the resale market, an optimal resale mechanism exists and can by computed using Myerson's (1981) methods as long as the resale seller's use value is commonly known and the beliefs about the other bidders are represented by smooth distributions.
} 
fully rational concerning the choice of a resale mechanism if any mechanism can be costlessly implemented.

The reason that Assumption 4 is sufficient for our results is that in the resale environments that are relevant for our equilibrium constructions, any standard auction with an optimal reserve price is an optimal resale mechanism. Other resale environments become relevant if a bidder deviates from her equilibrium bid in an attempt to win the auction more often and subsequently offer the good for resale, but we will show that even if the deviating bidder appropriates the entire surplus in the resale market no such deviation is profitable.

\section{Equilibrium resale environments}

The following two classes of resale environments are relevant for our equilibrium constructions. (i) The resale environments where the resale seller is certain about the maximum use value among all bidders. (ii) The resale environments where the resale seller is the speculator and, according to the post-auction beliefs, the $n$ regular bidders have independent private values, where each bidder's value is distributed according to $\hat{F}_{[0, \hat{\theta}]}$ for some $\hat{\theta} \in(0,1]$.

In class (i), any standard auction with a reserve price equal to the maximum use value is an optimal resale mechanism because the resale seller appropriates the entire surplus. The equilibrium resale allocation is that the resale seller keeps the good if she has the maximum use value, and otherwise sells it to the bidder with the maximum use value. In class (ii), it is well-known (cf. Myerson, 1981) that any standard auction with reserve price $\hat{r}(\hat{\theta})$ implicitly defined by $\hat{r}(\hat{\theta})=(F(\hat{\theta})-F(\hat{r}(\hat{\theta}))) / f(\hat{r}(\hat{\theta}))$ is an optimal resale mechanism (observe that the function $\hat{r}$ is uniquely determined on $(0,1]$, and is strictly increasing and continuous). The equilibrium resale allocation is that the bidder with the highest value obtains the good, unless her value is below $\hat{r}(\hat{\theta})$, in which case the resale seller keeps the good. In both classes (i) and (ii) of resale environments, we use the notation $\mathcal{S}(\check{r})$ to denote any standard auction with any reserve price $\check{r} \geq 0$.

Throughout the rest of this section, we consider the resale environments of class (ii). We derive auxiliary technical results about the resale mechanism $\mathcal{S}(\hat{r}(\hat{\theta}))$ for all $\hat{\theta} \in(0,1]$. 
Denote by $\hat{P}_{\hat{\theta}}(\theta)$ the expected payment of a regular bidder with use value $\theta \in[0,1]$ in the mechanism $\mathcal{S}(\hat{r}(\hat{\theta}))$, and by $\hat{Q}_{\hat{\theta}}(\theta)$ the probability that the bidder obtains the good. ${ }^{5}$ Properties of the functions $\hat{P}$ and $\hat{Q}$ are summarized in the following lemma.

Lemma 1 Consider any $\hat{\theta} \in(0,1]$. For all $\theta \in[0, \hat{r}(\hat{\theta}))$, we have $\hat{P}_{\hat{\theta}}(\theta)=0$ and $\hat{Q}_{\hat{\theta}}(\theta)=0$. For all $\theta \in[\hat{r}(\hat{\theta}), \hat{\theta}]$,

$$
\begin{aligned}
& \frac{\hat{P}_{\hat{\theta}}(\theta)}{\hat{Q}_{\hat{\theta}}(\theta)}=\theta-\int_{\hat{r}(\hat{\theta})}^{\theta} \frac{F\left(\theta^{\prime}\right)^{n-1}}{F(\theta)^{n-1}} d \theta^{\prime}, \\
& \frac{\hat{P}_{\hat{\theta}}(\theta)}{\hat{Q}_{\hat{\theta}}(\theta)} \text { is weakly increasing in } \theta \text { and } \hat{\theta} \\
& \hat{Q}_{\hat{\theta}}(\theta)=F(\theta)^{n-1} / F(\hat{\theta})^{n-1} \text {. }
\end{aligned}
$$

For all $\theta \in[\hat{\theta}, 1]$,

$$
\hat{Q}_{\hat{\theta}}(\theta)=1, \quad \hat{P}_{\hat{\theta}}(\theta)=\hat{P}_{\hat{\theta}}(\hat{\theta}) .
$$

Proof. Formulas (1), (3), and (4) are standard. To see (2), use that $\hat{r}$ is increasing and the derivative of $\hat{P}_{\hat{\theta}}(\theta) / \hat{Q}_{\hat{\theta}}(\theta)$ with respect to $\theta$ is non-negative.

QED

The resale seller's expected revenue in the mechanism $\mathcal{S}(\hat{r}(\hat{\theta}))$ is denoted $M(\hat{\theta})$; also let $M(0)=0$. Observe that $M$ is strictly increasing. Other properties of $M$ are summarized in the following lemma.

LEMMA 2 For all $\hat{\theta} \in(0,1]$,

$$
\begin{aligned}
M(\hat{\theta}) & =\int_{\hat{r}(\hat{\theta})}^{\hat{\theta}}\left(\theta-\frac{F(\hat{\theta})-F(\theta)}{f(\theta)}\right) \frac{d\left(F(\theta)^{n}\right)}{F(\hat{\theta})^{n}}, \\
M^{\prime}(\hat{\theta}) & =n \frac{f(\hat{\theta})}{F(\hat{\theta})}\left(\hat{\theta}-M(\hat{\theta})-\int_{\hat{r}(\hat{\theta})} \frac{F(\theta)^{n-1}}{F(\hat{\theta})^{n-1}} d \theta\right) .
\end{aligned}
$$

Moreover,

$$
M^{\prime}(0)<\frac{n-1}{n}
$$

The function $M$ is Lipschitz continuous on [0,1], and its derivative $M^{\prime}$ is continuous.

\footnotetext{
${ }^{5}$ Observe that according to the post-auction beliefs the probability equals 0 that a bidder-type in $(\hat{\theta}, 1]$ participates in the resale market. It is nevertheless important to define the functions $\hat{P}_{\hat{\theta}}(\theta)$ and $\hat{Q}_{\hat{\theta}}(\theta)$ for $\theta>\hat{\theta}$ because a participation of a type $\theta>\hat{\theta}$ may arise from a deviation in period 1 .
} 
Proof. Formula (5) is standard from Myerson (1981), while (6) follows from standard differentiation rules. By differentiability, $F(\theta)=f(0) \theta+\mathcal{o}(\theta)$. Using this and $\hat{r}(\hat{\theta}) / \hat{\theta} \rightarrow 1 / 2$ as $\hat{\theta} \rightarrow 0,(5)$ can be used to show

$$
M^{\prime}(0)=\lim _{\hat{\theta} \rightarrow 0} \frac{M(\hat{\theta})}{\hat{\theta}}=\frac{n-1}{n+1}+\frac{1}{2^{n}(n+1)},
$$

which implies (7). Using (6) and (8), it can be confirmed that $\lim _{\hat{\theta} \rightarrow 0} M^{\prime}(\hat{\theta})=M^{\prime}(0)$. Therefore, $M^{\prime}$ is continuous on $[0,1]$. Hence, $M^{\prime}$ is bounded above on $[0,1]$, which implies Lipschitz continuity of $M$.

$Q E D$

The final result in this section shows that the payment of the highest type of bidder who participates in the resale market is higher than the resale seller's expected revenue.

LEMMA 3

$$
\forall \hat{\theta} \in(0,1]: \hat{P}_{\hat{\theta}}(\hat{\theta})>M(\hat{\theta})
$$

Proof. Using Lemma 1, we find

$$
\begin{aligned}
& M(\hat{\theta})=n \int_{\hat{r}(\hat{\theta})}^{\hat{\theta}} \hat{P}_{\hat{\theta}}(\theta) \frac{\mathrm{d} F(\theta)}{F(\hat{\theta})}=n \int_{\hat{r}(\hat{\theta})}^{\hat{\theta}} \frac{\hat{P}_{\hat{\theta}}(\theta)}{\hat{Q}_{\hat{\theta}}(\theta)} \hat{Q}_{\hat{\theta}}(\theta) \frac{\mathrm{d} F(\theta)}{F(\hat{\theta})} \\
& \stackrel{(3)}{=} \int_{\hat{r}(\hat{\theta})}^{\hat{\theta}} \frac{\hat{P}_{\hat{\theta}}(\theta)}{\hat{Q}_{\hat{\theta}}(\theta)} \frac{\mathrm{d} F(\theta)^{n}}{F(\hat{\theta})^{n}} \stackrel{(2)}{\leq} \int_{\hat{r}(\hat{\theta})}^{\hat{\theta}} \frac{\hat{P}_{\hat{\theta}}(\hat{\theta})}{\hat{Q}_{\hat{\theta}}(\hat{\theta})} \frac{\mathrm{d} F(\theta)^{n}}{F(\hat{\theta})^{n}} \\
& \stackrel{(4)}{=} \hat{P}_{\hat{\theta}}(\hat{\theta})\left(1-\frac{F(\hat{r}(\hat{\theta}))^{n}}{F(\hat{\theta})^{n}}\right)<\hat{P}_{\hat{\theta}}(\hat{\theta})
\end{aligned}
$$

because $\hat{r}(\hat{\theta})<\hat{\theta}$.

Observe that Lemma 3 would be trivial if $n=1$ because nobody would pay more than the highest participating type. If $n>1$, the expected payment of any given bidder type is collected $n$ times if all resale bidders have this type. The proof instead works with any given type's expected payment divided by her winning probability. Because this divided payment is increasing in type and because with positive probability nobody wins, the highest type's divided payment exceeds the seller's revenue. But the highest type wins for sure, which completes the proof. 


\section{The first-price/Dutch auction with resale}

In this section we construct and discuss the essentially unique perfect Bayesian equilibrium of a first-price auction with resale. Proposition 1 describes the equilibrium. Proposition 2 deals with the question for which parameter constellations the speculator plays an active role. Proposition 3 evaluates the impact of a resale opportunity on initial seller revenue.

Let $\beta$ denote bidder $i$ 's $(i \in I)$ bid in the first-price auction as a function of her use value. Let $H$ denote the probability distribution for the speculator's bid $\tilde{b}_{s}$.

To define post-auction beliefs, consider any bidder $j \in I$. Let $i \in I \cup\{s\}$ denote the label of the winner, and let $b_{i} \geq 0$ denote the winner's bid. Then, the probability distribution $\Pi_{j}\left(\cdot \mid i, b_{i}\right)$ denotes the post-auction belief about $j$ 's use value held by bidders other than $j$.

Let $\mathcal{M}\left(i, b_{i}, \theta_{i}\right)$ denote the resale mechanism used by the resale seller $i \in I \cup\{s\}$ after a first-price auction when $b_{i} \geq 0$ denotes $i$ 's auction winning bid and $i$ 's use value is $\theta_{i}$. For all $i, j \in I \cup\{s\}$ with $j \neq i$, all $b_{i} \geq 0$, and all $\theta_{i}, \theta_{j}$, let $P_{j}\left(i, b_{i}, \theta_{i}, \theta_{j}\right)$ denote the net expected transfer from bidder $j$ of type $\theta_{j}$ to the other bidders (including the transfer to $i$ ) in the mechanism $\mathcal{M}\left(i, b_{i}, \theta_{i}\right)$. Let $Q_{j}\left(i, b_{i}, \theta_{i}, \theta_{j}\right)$ denote the probability that bidder $j$ obtains the good. Let $P\left(i, b_{i}, \theta_{i}\right)$ denote the expected transfer to the resale seller $i$, and $Q\left(i, b_{i}, \theta_{i}\right)$ the probability that the resale seller keeps the good.

For all $i \in I$, bidder $i$ 's expected payoff when she bids $b_{i} \geq 0$ and has the use value $\theta_{i}$ equals

$$
\begin{aligned}
u_{i}\left(b_{i}, \theta_{i}\right)= & E[ \\
& \left(-b_{i}+\max \left\{\theta_{i}, \delta\left(\theta_{i} Q\left(i, b_{i}, \theta_{i}\right)+P\left(i, b_{i}, \theta_{i}\right)\right)\right\}\right) \mathbf{1}_{w\left(b_{i}, \tilde{\mathbf{b}}_{-i}\right)=i} \\
& \left.+\sum_{j \neq i} \delta\left(\theta_{i} Q_{i}\left(j, \tilde{b}_{j}, \tilde{\theta}_{j}, \theta_{i}\right)-P_{i}\left(j, \tilde{b}_{j}, \tilde{\theta}_{j}, \theta_{i}\right)\right) \mathbf{1}_{w\left(b_{i}, \tilde{\mathbf{b}}_{-i}\right)=j}\right]
\end{aligned}
$$

where $w$ denotes the period-1 winner as a function of the bid profile, and where the max-term reflects the condition that after winning in period 1 bidder $i$ decides optimally whether to consume the good or to offer it for resale. The speculator's payoff when she bids $b_{s} \geq 0$ is given by

$$
u_{s}\left(b_{s}\right)=E\left[\left(-b_{s}+\delta P\left(s, b_{s}, \theta_{s}\right)\right) \mathbf{1}_{w\left(b_{s}, \tilde{\mathbf{b}}_{-s}\right)=s}\right]
$$


The equilibrium conditions are (10) that post-auction beliefs about auction losers are determined by Bayes rule whenever possible, (11) that the resale mechanism is chosen according to Assumption 4, and $(12,13)$ that period-1 behavior is optimal.

Definition 1 A tuple $(\beta, H, \mathcal{M})$ is a quasi-symmetric regular equilibrium of the first-price auction with resale if there exists a belief system $\left(\Pi_{j}\left(\cdot \mid i, b_{i}\right)\right)_{j \in I, i \in I \cup\{s\}, b_{i} \geq 0}$ such that the following holds:

$$
\begin{gathered}
\forall i \in I \cup\{s\}, b_{i}>0, j \in I \backslash\{i\}: \\
\Pi_{j}\left(\cdot \mid i, b_{i}\right)=\hat{F}_{\beta^{-1}\left(\left[0, b_{i}\right]\right)} \quad \text { if }\left[0, b_{i}\right] \cap \beta([0,1]) \neq \emptyset, \\
\forall i \in I \cup\{s\}, b_{i} \geq 0, \theta_{i}: \mathcal{M}\left(i, b_{i}, \theta_{i}\right)=\hat{\mathcal{M}}\left(\left(\Pi_{j}\left(\cdot \mid i, b_{i}\right)\right)_{j \in I}, \theta_{i}, i\right), \\
\forall i \in I, \theta_{i}: \beta\left(\theta_{i}\right) \in \arg \max _{b_{i} \geq 0} u_{i}\left(b_{i}, \theta_{i}\right), \\
\operatorname{Pr}\left[\tilde{b}_{s} \in \arg \max _{b_{s} \geq 0} u_{s}\left(b_{s}\right)\right]=1 .
\end{gathered}
$$

This equilibrium concept is in the spirit of perfect Bayesian equilibrium, combined with the symmetry and regularity restrictions formulated in Assumption 2, and with two presentational simplifications. First, we have omitted a condition on the post-auction beliefs about the auction winner because it would play no role for our analysis. Second, condition (10) excludes the case $b_{i}=0$; the appropriate Bayesian updating condition for this case would depend on the auction tieing rule (whereas, by Assumption 2, ties occur with probability 0 if $b_{i}>0$ ), but the case $b_{i}=0$ is irrelevant for our analysis because it will turn out that in equilibrium only positive bids can win the auction with positive probability.

Additional notation is needed in order to state the main result, Proposition 1, which describes the equilibrium. For all $\theta \in(0,1]$ and $b \in \mathbb{R}$, define

$$
K(\theta, b)= \begin{cases}N(\theta, b) & \text { if } b>\delta M(\theta), \\ \max \left\{\delta M^{\prime}(\theta), N(\theta, b)\right\} & \text { if } b \leq \delta M(\theta)\end{cases}
$$

where

$$
N(\theta, b)=(n-1) \frac{f(\theta)}{F(\theta)}(\theta-b) .
$$


Observe that in a first-price auction with $n$ regular bidders and without resale opportunity, $N(\theta, b)$ is the slope of the equilibrium bid function at $\theta$ when $b$ equals type $\theta$ 's equilibrium bid. Hence, $N(\theta, b)$ represents the competition among the regular bidders in the absence of resale. The function $K(\theta, b)$ equals $N(\theta, b)$ if $b>\delta M(\theta)$; that is, if $b$ and $\theta$ are such that, if resale is possible, a speculator who comes in with a bid of $b$ and wins against the regular-bidder types up to $\theta$ makes a loss. If type $\theta$ 's bid $b$ is such that the speculator breaks even $(b=\delta M(\theta))$, then $K(\theta, b)$ is the larger of $N(\theta, b)$ and the slope $\delta M^{\prime}(\theta)$ that keeps the speculator at the break-even point. In summary, $K(\theta, b)$ is the smallest slope that respects the competition among the regular bidders without resale and does not allow the speculator to make a profit via resale.

For any $\bar{b}>0$, consider a strictly increasing Lipschitz continuous function $\phi:[0, \bar{b}] \rightarrow[0,1]$ such that $\phi(b)>b \geq \delta M(\phi(b))$ for all $b>0$. For all $b \in(0, \bar{b}]$ where the derivative $\phi^{\prime}(b)$ exists, define

$$
L_{\phi}(b)= \begin{cases}\frac{R_{\phi}(b)}{S_{\phi}(b)} & \text { if } b=\delta M(\phi(b)) \\ 0 & \text { if } b>\delta M(\phi(b))\end{cases}
$$

where

$$
\begin{aligned}
R_{\phi}(b) & =1-N(\phi(b), b) \phi^{\prime}(b) \\
S_{\phi}(b) & =(1-\delta) \phi(b)-b+\delta \hat{P}_{\phi(b)}(\phi(b)) .
\end{aligned}
$$

Observe that $L_{\phi}$ is well-defined because $b=\delta M(\phi(b))$ implies $S_{\phi}(b)>0$ by (9). Also observe that $L_{\phi}(b)=0$ if $b$ is such that the speculator would make a loss if she did bid $b$ and did win against the types in $[0, \phi(b)]$.

Proposition 1 describes the unique equilibrium. ${ }^{6}$ Condition (18) establishes a differential equation for the regular bidders' bid function $\beta$. The differential equation, which holds almost everywhere according to the Lebesgue measure, reflects the bidding competition among the

\footnotetext{
${ }^{6}$ The uniqueness property differs from the "essential uniqueness" in the Note where multiple optimal resale prices cannot be excluded because value distributions $F$ with a non-increasing hazard rate are allowed; cf. footnote 1 in the Note and p. 7 in the Note.
} 
regular bidders and a no-profit condition for the speculator (recall the properties of the function $K$ explained above). The speculator randomizes her bid according to (19), in which the distribution $H$ is constructed such that it is optimal for the regular bidders to use the bid function defined by (18). Using (16) one sees that the support of $H$ is confined to the points $\theta$ where $\beta(\theta)=\delta M(\theta)$ so that the speculator does not make a loss. ${ }^{7}$ The post-auction beliefs (20) of the winning bidder are that the highest type among the losing regular bidders is the type that would have resulted in a tie. Hence, if one of the regular bidders wins the auction after making her equilibrium bid, she believes that she has the highest use value in the market and thus consumes the good. Condition (21) states that if the auction winner is the speculator, then her resale mechanism is a standard auction with a reserve price that is optimal given her post-auction beliefs.

Proposition 1 For any regular-bidder number $n \geq 2$, discount factor $\delta \in(0,1)$, and distribution F satisfying Assumption 1, the first-price auction with resale has a unique quasisymmetric regular equilibrium $(\beta, H, \mathcal{M})$. The equilibrium satisfies conditions (18) (19), and (21). It is supported by beliefs satisfying (20).

$$
\begin{gathered}
\beta \text { is Lipschitz, } \beta(0)=0, \beta^{\prime}(\theta)=K(\theta, \beta(\theta)) \text { a.e. } \theta \in(0,1) . \\
\forall b \in(0, \beta(1)]: H(b)=e^{-\int_{b}^{\beta(1)} L_{\phi}(b) d b}
\end{gathered}
$$

where $\phi$ denotes the inverse of $\beta$.

$$
\forall i, b_{i} \geq 0, j \notin\{s, i\}, \theta_{j}: \Pi_{j}\left(\theta_{j} \mid i, b_{i}\right)=\min \left\{\frac{F\left(\theta_{j}\right)}{F\left(\phi\left(b_{i}\right)\right)}, 1\right\},
$$

where we define $\phi\left(b_{i}\right)=1$ for all $b_{i}>\beta(1)$.

$$
\forall b_{s}>0: \mathcal{M}\left(s, b_{s}, 0\right)=\mathcal{S}\left(\hat{r}\left(\phi\left(b_{s}\right)\right)\right) .
$$

\footnotetext{
${ }^{7}$ The feature that the regular bidders use a strictly increasing bid function while an additional bidder with no private information randomizes over a subset of the regular bidders' bid range also appears in Martinez (2002). She constructs an equilibrium for first-price auctions without resale in an environment with three or more regular bidders and one additional bidder with a commonly known valuation. The differences between Martinez' equilibrium and ours are parallel to the differences between Vickrey's (1961, Appendix III) equilibrium and our equilibrium in the 2-bidder case; cf. footnote 6 in the Note.
} 
Proof. We first show uniqueness (Lemma 4 to Lemma 11), then existence (Lemma 12 to Lemma 15).

Lemma 4 shows that no regular bidder will bid above her value. Lemma 5 establishes a convenient representation for the speculator's payoff function. Lemma 6 shows that, with positive probability, the speculator makes arbitrarily small bids. Lemma 7 shows that the speculator's equilibrium payoff equals 0. Lemma 8 shows that the speculator's bid distribution has no atoms. Lemma 9 states that the regular bidders' equilibrium bid function is continuous. Lemma 10 states that every regular bidder with a positive use value will bid lower than her use value. Lemma 11 shows that the regular bidders' equilibrium bid function satisfies (18) and is uniquely characterized by (18), and the speculator's bid distribution is given by (19). Conditions (20) are (21) are then straightforward. This completes the equilibrium uniqueness proof.

In Lemma 12 and Lemma 14, we apply techniques from the theory of differential inclusions $^{8}$ to show that the differential equation (18) has a solution. In doing this, we also recall in Lemma 13 the differential equation satisfied by the standard no-resale first-price auction equilibrium bid function. Lemma 15 completes the equilibrium existence proof.

For any bid function $\beta$ satisfying Assumption 2 and all $b \in[0, \beta(1)]$, let

$$
\phi(b)=\sup \left\{\theta_{i} \in[0,1] \mid \beta\left(\theta_{i}\right)<b\right\},
$$

where $\sup \emptyset=0$.

LEMMA 4 Let $(\beta, H, \mathcal{M})$ be a quasi-symmetric regular equilibrium of the first-price auction with resale, and let $\phi$ be defined by (22). Then $\beta\left(\theta_{i}\right) \leq \theta_{i}$ for all $\theta_{i} \in[0,1]$, and $\phi(b) \geq b$ for all $b \in[0, \beta(1)]$.

Proof. By Assumption 2, $\beta(0)=0$. Now suppose that $\beta\left(\theta_{i}\right)>\theta_{i}$ for some $\theta_{i}>0$. In particular, $\beta\left(\theta_{i}\right)>0$ and thus $\beta\left(\theta_{i}\right)$ wins with positive probability by Assumption 2 .

\footnotetext{
${ }^{8}$ We thank Jörg Oechssler for help with this part of the proof.
} 
Therefore, $H\left(\beta\left(\theta_{i}\right)\right)>0$ and $F\left(\phi\left(\beta\left(\theta_{i}\right)\right)\right)^{n-1}>0$. A deviation to the bid $b_{i}=\theta_{i}$ is profitable because

$$
\begin{aligned}
& u_{i}\left(\theta_{i}, \theta_{i}\right)-u_{i}\left(\beta\left(\theta_{i}\right), \theta_{i}\right) \\
& =H\left(\beta\left(\theta_{i}\right)\right) F\left(\phi\left(\beta\left(\theta_{i}\right)\right)\right)^{n-1}\left(\beta\left(\theta_{i}\right)-\theta_{i}\right) \\
& \quad+\delta E[\underbrace{\left(\theta_{i} \hat{Q}_{\phi\left(\tilde{b}_{s}\right)}\left(\theta_{i}\right)-\hat{P}_{\phi\left(\tilde{b}_{s}\right)}\left(\theta_{i}\right)\right)}_{\geq 0} \mathbf{1}_{\tilde{b}_{s}>\tilde{b}_{-s-i}^{(1)}, \theta_{i}<\tilde{b}_{s} \leq \beta\left(\theta_{i}\right)}] \\
& >0 .
\end{aligned}
$$

To prove the second part of the lemma, fix $b>0$ and consider any $\theta_{i}<b$. Then $\beta\left(\theta_{i}\right) \leq$ $\theta_{i}<b$. Because $\theta_{i}$ is arbitrary, $\phi(b) \geq b$.

LEMma 5 Let $(\beta, H, \mathcal{M})$ be a quasi-symmetric regular equilibrium of the first-price auction with resale, and let $\phi$ be defined by (22). Then,

$$
\forall b>0, H(b)>0: u_{s}(b)=F(\phi(b))^{n}(\delta M(\phi(b))-b) .
$$

Proof. Consider any $b>0$ such that $H(b)>0$ and any $\theta^{\prime}<\phi(b)$. It follows $\beta\left(\theta^{\prime}\right)<b$ (otherwise $\beta(\theta) \geq b$ for all $\theta>\theta^{\prime}$ by Assumption 2, implying $\phi(b) \leq \theta^{\prime}$ ). Hence, the set $\{\theta \in[0,1] \mid \beta(\theta)<b\}$ equals $[0, \phi(b))$ or $[0, \phi(b)]$.

$Q E D$

The following lemma shows that bidder 2 makes, with positive probability, arbitrarily small bids. The proof is similar to that of Lemma 1 in the Note. One supposes that the speculator's infimum equilibrium bid $\underline{b}>0$. Then low regular-bidder types never win the auction. For some of these types it is profitable to deviate to a bid slightly above $\underline{b}$ because, combining (9) with a continuity argument, the deviating bid is smaller than the speculator's resale price.

LEMMA 6 Let $(\beta, H, \mathcal{M})$ be a quasi-symmetric regular equilibrium of the first-price auction with resale. Then $H(b)>0$ for all $b>0$. 
Proof. Let $\underline{b}=\inf \{b \mid H(b)>0\}$. Suppose that $\underline{b}>0$. Let $U_{s} \geq 0$ denote the speculator's equilibrium payoff. First consider the case $H(\underline{b})=0$ (i.e., no atom at $\underline{b}$ ). Then there exists a sequence $\left(b^{m}\right)_{m \in \mathbb{N}}$ such that $b^{m} \rightarrow \underline{b}$ as $m \rightarrow \infty, u_{s}\left(b^{m}\right)=U_{s}$, and $b^{m}>\underline{b}$ for all $m$. By Lemma 5,

$$
\forall m: U_{s}=F\left(\phi\left(b^{m}\right)\right)^{n}\left(\delta M\left(\phi\left(b^{m}\right)\right)-b^{m}\right) .
$$

By Lemma $4, \phi\left(b^{m}\right)>0$, hence (23) implies $\delta M\left(\phi\left(b^{m}\right)\right)-b^{m} \geq 0$. Defining $\underline{\theta}=\lim _{m} \phi\left(b^{m}\right)$, it follows $\delta M(\underline{\theta}) \geq \underline{b}$. This together with (9) implies

$$
\exists \xi>0: \quad \hat{P}_{\underline{\theta}}(\underline{\theta}) \geq M(\underline{\theta})+\xi \geq \frac{\underline{b}}{\delta}+\xi .
$$

Continuity of $\hat{P}$ by Lemma 1 together with (24) implies that there exists $b^{\prime}>\underline{b}$ and $\theta^{\prime}<\underline{\theta}$ such that

$$
\forall b \in\left(\underline{b}, b^{\prime}\right): \hat{P}_{\phi(b)}\left(\theta^{\prime}\right) \geq \frac{\underline{b}}{\delta}+\frac{\xi}{2} .
$$

Now consider any bidder $i \in I$ with type $\theta_{i}=\theta^{\prime}$. By construction, her equilibrium bid $b_{i}=\beta\left(\theta^{\prime}\right)$ never wins. Moreover, if some bidder $j \in I \backslash\{i\}$ wins then $b_{j}=\beta\left(\theta_{j}\right)>\underline{b} \geq b_{i}$, implying $\theta_{j}>\theta^{\prime}$; i.e., bidder $j$ will not resell to bidder $i$. Therefore, $Q_{i}\left(j, b_{j}, \theta_{j}, \theta^{\prime}\right)=0$ and $P_{i}\left(j, b_{j}, \theta_{j}, \theta^{\prime}\right)=0$. Therefore,

$$
u_{i}\left(\beta\left(\theta^{\prime}\right), \theta^{\prime}\right)=\delta E\left[\left(\theta^{\prime} \hat{Q}_{\phi\left(\tilde{b}_{s}\right)}\left(\theta^{\prime}\right)-\hat{P}_{\phi\left(\tilde{b}_{s}\right)}\left(\theta^{\prime}\right)\right) \boldsymbol{1}_{\tilde{b}_{s}>\tilde{b}_{-s-i}^{(1)}}\right] .
$$

On the other hand, for all $b_{i}>\underline{b}$,

$$
\begin{aligned}
u_{i}\left(b_{i}, \theta^{\prime}\right) \geq & \delta E\left[\left(\theta^{\prime} \hat{Q}_{\phi\left(\tilde{b}_{s}\right)}\left(\theta^{\prime}\right)-\hat{P}_{\phi\left(\tilde{b}_{s}\right)}\left(\theta^{\prime}\right)\right) \boldsymbol{1}_{\tilde{b}_{s}>\tilde{b}_{-s-i}^{(1)}, \tilde{b}_{s}>b_{i}}\right] \\
& +\left(\theta^{\prime}-b_{i}\right) \operatorname{Pr}\left[\tilde{b}_{s}>\tilde{b}_{-s-i}^{(1)}, \tilde{b}_{s} \leq b_{i}\right] .
\end{aligned}
$$

Therefore, for all $b_{i} \in\left(\underline{b}, b^{\prime}\right)$,

$$
\begin{aligned}
u_{i}\left(b_{i}, \theta^{\prime}\right)-u_{i}\left(\beta\left(\theta^{\prime}\right), \theta^{\prime}\right) \geq & -\delta E\left[\left(\theta^{\prime} \hat{Q}_{\phi\left(\tilde{b}_{s}\right)}\left(\theta^{\prime}\right)-\hat{P}_{\phi\left(\tilde{b}_{s}\right)}\left(\theta^{\prime}\right)\right) \mathbf{1}_{\tilde{b}_{s}>\tilde{b}_{-s-i}^{(1)}, \tilde{b}_{s} \leq b_{i}}\right] \\
& +\left(\theta^{\prime}-b_{i}\right) \operatorname{Pr}\left[\tilde{b}_{s}>\tilde{b}_{-s-i}^{(1)}, \tilde{b}_{s} \leq b_{i}\right] \\
\geq & E\left[\left(\delta \hat{P}_{\phi\left(\tilde{b}_{s}\right)}\left(\theta^{\prime}\right)-b_{i}\right) \mathbf{1}_{\left.\tilde{b}_{s}>\tilde{b}_{-s-i}^{(1)}, \tilde{b}_{s} \leq b_{i}\right]}\right] \\
\stackrel{(25)}{\geq} & E\left[\left(\underline{b}+\frac{\delta \xi}{2}-b_{i}\right) \mathbf{1}_{\tilde{b}_{s}>\tilde{b}_{-s-i}^{(1)}, \tilde{b}_{s} \leq b_{i}}\right] \\
= & \left(\underline{b}+\frac{\delta \xi}{2}-b_{i}\right) \underbrace{\operatorname{Pr}\left[\tilde{b}_{s}>\tilde{b}_{-s-i}^{(1)}, \tilde{b}_{s} \leq b_{i}\right]}_{\geq \operatorname{Pr}\left[\tilde{\theta}_{-i}^{(1)<\theta}, \tilde{b}_{s} \leq b_{i}\right]>0} .
\end{aligned}
$$


Hence, $u_{i}\left(b_{i}, \theta^{\prime}\right)>u_{i}\left(\beta\left(\theta^{\prime}\right), \theta^{\prime}\right)$ for all $b_{i}>\underline{b}$ that are sufficiently close to $\underline{b}$. This contradicts (12).

In the case $H(\underline{b})>0$ the proof is similar. One defines $\underline{\theta}=\phi(\underline{b})$ and shows that the deviation $b_{1}=\underline{b}$ is profitable for some type $\theta^{\prime}<\underline{\theta}$.

QED

LEMMA 7 In any quasi-symmetric regular equilibrium of the first-price auction with resale, the speculator's payoff equals 0.

Proof. Let $U_{s} \geq 0$ denote the speculator's equilibrium payoff. Suppose that $U_{s}>0$. This implies $H(0)=0$. Hence, by Lemma 6 there exists a sequence $\left(b^{m}\right)_{m \in \mathbb{N}}$ such that $b^{m} \rightarrow 0$ as $m \rightarrow \infty, u_{s}\left(b^{m}\right)=U_{s}$, and $b^{m}>0$ for all $m$. By Lemma 5,

$$
\forall m: U_{s}=F\left(\phi\left(b^{m}\right)\right)^{n}\left(\delta M\left(\phi\left(b^{m}\right)\right)-b^{m}\right) \leq M\left(\phi\left(b^{m}\right)\right)
$$

Hence, $M(\underline{\theta}) \geq U_{s}>0$, where $\underline{\theta} \equiv \lim _{m} \phi\left(b^{m}\right)$. Note that

$$
\forall \theta<\underline{\theta}: \beta(\theta)=0
$$

By $(9), \hat{P}_{\underline{\theta}}(\underline{\theta})>U_{s}$. As in the proof of Lemma 6 , continuity of $\hat{P}$ by Lemma 1 now shows the existence of $b^{\prime}>0$ and $\theta^{\prime}<\underline{\theta}$ such that

$$
\forall b \in\left(0, b^{\prime}\right): \hat{P}_{\phi(b)}\left(\theta^{\prime}\right) \geq \frac{U_{s}}{2} .
$$

Consider a bidder $i \in I$ with type $\theta_{i}=\theta^{\prime}$. By (26), her equilibrium bid $\beta\left(\theta^{\prime}\right)=0$ never wins. In the same manner as in the proof of Lemma 6, one obtains a contradiction by showing that a deviation to a small positive bid $b_{i}>0$ is profitable.

QED

LEMma 8 Let $(\beta, H, \mathcal{M})$ be a quasi-symmetric regular equilibrium of the first-price auction with resale. Then $H$ is continuous on $(0, \infty)$.

Proof. Suppose that there exists $b^{\prime}>0$ where $H$ is not continuous; i.e., $\operatorname{Pr}\left[\tilde{b}_{s}=b^{\prime}\right]>0$. 
Define $\theta^{m}=\phi\left(b^{\prime}\right)-1 / m$ for all $m$ large enough such that $\theta^{m}>0$. Let $\bar{b}=\lim _{m \rightarrow \infty} \beta\left(\theta^{m}\right)$. We have $\bar{b}=b^{\prime}$ because otherwise $\operatorname{Pr}\left[\beta\left(\tilde{\theta}_{i}\right) \in\left(\bar{b}, b^{\prime}\right)\right]=0$ which would imply $u_{s}\left(\left(b^{\prime}+\bar{b}\right) / 2\right)>$ $u_{s}\left(b^{\prime}\right)$.

By (9) there exists $\xi>0$ such that $\hat{P}_{\phi\left(b^{\prime}\right)}\left(\phi\left(b^{\prime}\right)\right) \geq M\left(\phi\left(b^{\prime}\right)\right)+\xi \geq b^{\prime} / \delta+\xi$ because otherwise $u_{s}\left(b^{\prime}\right)<0$. By Lemma 1 , the function $\hat{P}_{\phi\left(b^{\prime}\right)}$ is continuous at $\phi\left(b^{\prime}\right)$ and thus

$$
\hat{P}_{\phi\left(b^{\prime}\right)}\left(\theta^{m}\right)>b^{\prime} / \delta+\xi / 2
$$

for all large $m$. Therefore, for large $m$,

$$
\begin{aligned}
& u_{i}\left(b^{\prime}, \theta^{m}\right)-u_{i}\left(\beta\left(\theta^{m}\right), \theta^{m}\right) \\
& \geq H\left(\beta\left(\theta^{m}\right)\right) F\left(\phi\left(\beta\left(\theta^{m}\right)\right)\right)^{n-1}\left(\beta\left(\theta^{m}\right)-b^{\prime}\right)+\operatorname{Pr}\left[\tilde{b}_{s} \in\left(\beta\left(\theta^{m}\right), b^{\prime}\right)\right](-1) \\
& \quad+\operatorname{Pr}\left[\tilde{b}_{s}=b^{\prime}\right] F\left(\phi\left(b^{\prime}\right)\right)^{n-1}\left((1-\delta) \theta^{m}+\left(\delta \hat{P}_{\phi\left(b^{\prime}\right)}\left(\theta^{m}\right)-b^{\prime}\right)\right) .
\end{aligned}
$$

Therefore,

$$
\begin{aligned}
& \lim \inf _{m \rightarrow \infty} u_{1}\left(b^{\prime}, \theta^{m}\right)-u_{1}\left(b_{1}\left(\theta^{m}\right), \theta^{m}\right) \\
& \left.\quad \geq \operatorname{Pr}\left[\tilde{b}_{s}=b^{\prime}\right] F\left(\phi\left(b^{\prime}\right)\right)^{n-1}\left(\delta \hat{P}_{\phi\left(b^{\prime}\right)}\left(\theta^{m}\right)-b^{\prime}\right)\right)>0 .
\end{aligned}
$$

I.e., for large $m$ type $\theta^{m}$ has a profitable deviation.

LEMma 9 Let $(\beta, H, \mathcal{M})$ be a quasi-symmetric regular equilibrium of the first-price auction with resale, and let $\phi$ be defined by (22). Then $\beta$ is continuous. Moreover, $\phi\left(\beta\left(\theta_{i}\right)\right)=\theta_{i}$ for all $\theta_{i} \in[0,1]$. The function $\phi$ is continuous and strictly increasing.

Proof. Standard.

LEMMA 10 Let $(\beta, H, \mathcal{M})$ be a quasi-symmetric regular equilibrium of the first-price auction with resale, and let $\phi$ be defined by (22). Then $\beta\left(\theta_{i}\right)<\theta_{i}$ for all $\theta_{i} \in(0,1]$. Moreover, $\phi(b)>b$ for all $b \in(0, \beta(1)]$. 
Proof. Suppose that $\beta\left(\theta_{i}\right) \geq \theta_{i}>0$. Then $\beta\left(\theta_{i}\right)>0$, implying $H\left(\beta\left(\theta_{i}\right)\right)>0$ by Lemma 6 . Now Lemma 4 shows that $\beta\left(\theta_{i}\right)=\theta_{i}$. Finally, a computation similar to that in the proof of Lemma 4 shows that a deviation to the bid $b_{i}=\theta_{i} / 2$ is profitable - a contradiction.

The claim about $\phi$ now follows from Lemma 9.

The following lemma establishes the differential equation that characterizes the bid function $\beta$, and simultaneously determines the bid distribution $H$. The proof relies on local optimality of every regular-bidder type's bid, and on the result from Lemma 7 that the speculator's payoff equals 0 . Throughout the proof, we distinguish two kinds of bids $b \in(0, \beta(1)]$ : we write $\Xi(b)=0$ if $b$ has a neighborhood where the speculator bids with probability 0 , and write $\Xi(b)=1$ otherwise.

We begin by using upward optimality of the bid function $\beta$ to derive an inequality (29) in terms of $H$ and the inverse bid function $\phi$. Inequality (29) captures that the payoff increase effect from buying more often in the auction and less often from the speculator, weighted with the probability mass $H\left(b_{i}\right)-H\left(b_{i}^{\prime}\right)$, is offset by the payoff decrease effect from bidding higher and winning more often against one of the other regular bidders.

We continue by showing that $H$ is locally Lipschitz continuous on $(0, \beta(1)]$. Local Lipschitz continuity around any $b$ with $\Xi(b)=0$ is easy because $H$ is locally constant there. If $\Xi(b)=1$, then we use (9) and the result that the speculator obtains 0 payoff (31) to obtain a positive lower bound (32) for the payoff increase effect in (29). Because the payoff increase effect is weighted with the probability mass $H\left(b_{i}\right)-H\left(b_{i}^{\prime}\right)$, we can conclude that $H$ is locally Lipschitz around $b$.

The payoff decrease effect in (29) is confirmed in (33) and is then used to show in (34) that the slope of the inverse bid function $\phi$ is bounded above by the slope that would be relevant in the absence of a resale opportunity.

Next we use downward optimality of the bid function $\beta$ to derive an inequality (35) parallel to (29). Inequality (35) includes a payoff increase effect from bidding lower and winning less often against one of the other regular bidders, as confirmed in (36).

Combining the payoff decrease effect (33) with the payoff increase effect (36), we obtain 
the slope of the inverse bid function $\phi$ in the neighborhood of points where the speculator does not bid (37). Combining this with the 0-payoff condition for the speculator and with the upper bound (34) we obtain a differential equation (40) for $\phi$. Inverting this equation yields the differential equation (18) for $\beta$. Using that $K$ is strictly decreasing in its second argument, we show that the solution to (18) is unique.

Finally, we combine the inequalities (29) and (35) derived from upward and downward optimality of $\beta$ with the fact that $H$ is locally Lipschitz to obtain a differential equation for $H$, see (42). This equation has the unique solution (19).

LEMMA 11 Let $(\beta, H, \mathcal{M})$ be a quasi-symmetric regular equilibrium of the first-price auction with resale and $\phi$ be defined by (22). Then $\beta$ satisfies (18) and is uniquely determined by these conditions. The distribution $H$ is given by (19).

Proof. For all $i \in I, b_{i} \in(0, \beta(1)]$, and $\theta_{i} \in[0,1]$,

$$
\begin{aligned}
u_{i}\left(b_{i}, \theta_{i}\right)= & H\left(b_{i}\right) F\left(\phi\left(b_{i}\right)\right)^{n-1}\left(\theta_{i}-b_{i}\right) \\
& +\delta \int_{b_{i}}^{\infty} F\left(\phi\left(b_{s}\right)\right)^{n-1}\left(\theta_{i} \hat{Q}_{\phi\left(b_{s}\right)}\left(\theta_{i}\right)-\hat{P}_{\phi\left(b_{s}\right)}\left(\theta_{i}\right)\right) \mathrm{d} H\left(b_{s}\right) .
\end{aligned}
$$

Consider $b_{i}, b_{i}^{\prime} \in(0, \beta(1)]$ with $b_{i}^{\prime}<b_{i}$, and $\theta_{i}^{\prime}=\phi\left(b_{i}^{\prime}\right)$. Note that

$$
\begin{aligned}
& \delta \int_{b_{i}^{\prime}}^{b_{i}} F\left(\phi\left(b_{s}\right)\right)^{n-1} \underbrace{\hat{Q}_{\phi\left(b_{s}\right)}\left(\theta_{i}^{\prime}\right)}_{\text {decreasing in } b_{s}} \underbrace{\left(\theta_{i}^{\prime}-\frac{\hat{P}_{\phi\left(b_{s}\right)}\left(\theta_{i}^{\prime}\right)}{\hat{Q}_{\phi\left(b_{s}\right)}\left(\theta_{i}^{\prime}\right)}\right)}_{\text {decreasing in } b_{s} \text { by }(1)} \mathrm{d} H\left(b_{s}\right) \\
& \leq \delta F\left(\phi\left(b_{i}\right)\right)^{n-1}\left(\phi\left(b_{i}^{\prime}\right)-\hat{P}_{\phi\left(b_{i}^{\prime}\right)}\left(\phi\left(b_{i}^{\prime}\right)\right)\right)\left(H\left(b_{i}\right)-H\left(b_{i}^{\prime}\right)\right),
\end{aligned}
$$

because $\hat{Q}_{\phi\left(b_{i}^{\prime}\right)}\left(\phi\left(b_{i}^{\prime}\right)\right)=1$. Hence,

$$
\begin{aligned}
0 \stackrel{(12)}{\geq} & u_{i}\left(b_{i}, \theta_{i}^{\prime}\right)-u_{i}\left(b_{i}^{\prime}, \theta_{i}^{\prime}\right) \\
\stackrel{(27)}{=} & \left(H\left(b_{i}\right) F\left(\phi\left(b_{i}\right)\right)^{n-1}-H\left(b_{i}^{\prime}\right) F\left(\phi\left(b_{i}^{\prime}\right)\right)^{n-1}\right) \theta_{i}^{\prime} \\
& -\left(H\left(b_{i}\right) F\left(\phi\left(b_{i}\right)\right)^{n-1} b_{i}-H\left(b_{i}^{\prime}\right) F\left(\phi\left(b_{i}^{\prime}\right)\right)^{n-1} b_{i}^{\prime}\right) \\
& -\delta \int_{b_{i}^{\prime}}^{b_{i}} F\left(\phi\left(b_{s}\right)\right)^{n-1}\left(\hat{Q}_{\phi\left(b_{s}\right)}\left(\theta_{i}^{\prime}\right) \theta_{i}^{\prime}-\hat{P}_{\phi\left(b_{s}\right)}\left(\theta_{i}^{\prime}\right)\right) \mathrm{d} H\left(b_{s}\right) \\
& \quad-H\left(b_{i}^{\prime}\right) k_{1}\left(b_{i}, b_{i}^{\prime}\right)\left(b_{i}-b_{i}^{\prime}\right)+k_{2}\left(b_{i}, b_{i}^{\prime}\right)\left(H\left(b_{i}\right)-H\left(b_{i}^{\prime}\right)\right),
\end{aligned}
$$


where

$$
\begin{aligned}
& k_{1}\left(b_{i}, b_{i}^{\prime}\right)=F\left(\phi\left(b_{i}\right)\right)^{n-1}-\frac{F\left(\phi\left(b_{i}\right)\right)^{n-1}-F\left(\phi\left(b_{i}^{\prime}\right)\right)^{n-1}}{b_{i}-b_{i}^{\prime}}\left(\phi\left(b_{i}^{\prime}\right)-b_{i}^{\prime}\right), \\
& k_{2}\left(b_{i}, b_{i}^{\prime}\right)=F\left(\phi\left(b_{i}\right)\right)^{n-1}\left(\phi\left(b_{i}^{\prime}\right)(1-\delta)+\delta \hat{P}_{\phi\left(b_{i}^{\prime}\right)}\left(\phi\left(b_{i}^{\prime}\right)\right)-b_{i}\right) .
\end{aligned}
$$

Consider any $b \in(0, \beta(1)]$. We write $\Xi(b)=0$ if

$$
\exists \nu>0: \operatorname{Pr}\left[\tilde{b}_{s} \in(b-\nu, b+\nu)\right]=0,
$$

and $\Xi(b)=1$ otherwise. By Lemma 7 ,

$$
\text { if } \Xi(b)=1 \text { then } \exists\left(c^{m}\right), c^{m} \rightarrow b, c^{m} \neq b: \delta M\left(\phi\left(c^{m}\right)\right)=c^{m} .
$$

Therefore, by continuity of $M$ and $\phi$,

$$
\text { if } \Xi(b)=1 \text { then } M(\phi(b))=b \text {. }
$$

Suppose that $\Xi(b)=0$. Then, there exists $\nu>0$ such that in the neighborhood $N(b)=$ $(b-\nu, b+\nu) \cap(0, \beta(1)]$ of $b$ the function $H$ is constant. In particular, $H$ is Lipschitz in $N(b)$. Now suppose that $\Xi(b)=1$. Using $(9)$ and (31), there exists $\xi>0$ such that for all $b_{i}, b_{i}^{\prime}$ in some neighborhood of $b$, if $b_{i}^{\prime}<b_{i}$,

$$
\begin{aligned}
k_{2}\left(b_{i}, b_{i}^{\prime}\right) & \geq F\left(\phi\left(b_{i}\right)\right)^{n-1}\left(\phi\left(b_{i}^{\prime}\right)(1-\delta)+\delta M\left(\phi\left(b_{i}^{\prime}\right)\right)+\delta \xi-b_{i}\right) \\
& \geq F\left(\phi\left(b_{i}\right)\right)^{n-1} \frac{\delta \xi}{2} .
\end{aligned}
$$

Therefore,

$$
\begin{array}{r}
\forall b, \Xi(b)=1 \quad \exists \underline{k}(b)>0 \text { and a neighborhood } \mathcal{N}(b) \ni b: \\
\forall b_{i}, b_{i}^{\prime} \in \mathcal{N}(b), b_{i}^{\prime}<b_{i}: \quad k_{2}\left(b_{i}, b_{i}^{\prime}\right) \geq \underline{k}(b) .
\end{array}
$$

Together with $(29)$ and $k_{1}\left(b_{i}, b_{i}^{\prime}\right) \leq 1,(32)$ implies that $H$ is Lipschitz in $\mathcal{N}(b)$. We therefore conclude that in either case, $\Xi(b)=0$ or $\Xi(b)=1$, the function $H$ is Lipschitz in a neighborhood of $b$, proving that $H$ is locally Lipschitz in $(0, \beta(1)]$. In particular, $H$ is differentiable almost everywhere on $(0, \beta(1)]$. 
Next we show that

$$
\forall b_{i} \in(0, \beta(1)], b_{i}^{\prime} \in(0, \beta(1)], b_{i}^{\prime}<b_{i}: k_{1}\left(b_{i}, b_{i}^{\prime}\right) \geq 0
$$

Consider any $b \in(0, \beta(1)]$. If $\Xi(b)=0$ then $k_{1}\left(b_{i}, b_{i}^{\prime}\right) \geq 0$ holds for all $b_{i}, b_{i}^{\prime}$ in a neighborhood of $b$ because $H\left(b_{i}\right)=H\left(b_{i}^{\prime}\right)$. If $\Xi(b)=1$, then (32) together with (29) implies that $k_{1}\left(b_{i}, b_{i}^{\prime}\right) \geq$ 0 holds for all $b_{i}, b_{i}^{\prime}$ in a neighborhood of $b$. This completes the proof of (33).

Using the definition of $k_{1}$, (33) implies

$$
\forall b_{i} \in(0, \beta(1)), \phi \text { differentiable at } b_{i}: \phi^{\prime}\left(b_{i}\right) \leq \frac{1}{N\left(\phi\left(b_{i}\right), b_{i}\right)}
$$

Note that for all $b_{i}, b_{i}^{\prime} \in(0, \beta(1)]$ with $b_{i}^{\prime}<b_{i}$, and $\theta_{i}=\phi\left(b_{i}\right)$,

$$
\begin{aligned}
0 \leq & u_{i}\left(b_{i}, \theta_{i}\right)-u_{i}\left(b_{i}^{\prime}, \theta_{i}\right) \\
= & \left(H\left(b_{i}\right) F\left(\phi\left(b_{i}\right)\right)^{n-1}-H\left(b_{i}^{\prime}\right) F\left(\phi\left(b_{i}^{\prime}\right)\right)^{n-1}\right) \theta_{i} \\
& -\left(H\left(b_{i}\right) F\left(\phi\left(b_{i}\right)\right)^{n-1} b_{i}-H\left(b_{i}^{\prime}\right) F\left(\phi\left(b_{i}^{\prime}\right)\right)^{n-1} b_{i}^{\prime}\right) \\
& -\delta \int_{b_{i}^{\prime}}^{b_{i}} F\left(\phi\left(b_{s}\right)\right)^{n-1}(\underbrace{\hat{Q}_{\phi\left(b_{s}\right)}\left(\theta_{i}\right)}_{=1 \text { by }(4)} \theta_{i}-\underbrace{\hat{P}_{\phi\left(b_{s}\right)}\left(\theta_{i}\right)}_{=\hat{P}_{\theta_{i}}\left(\theta_{i}\right) \text { by }(4)}) \mathrm{d} H\left(b_{s}\right) \\
\leq & -H\left(b_{i}\right) l_{1}\left(b_{i}, b_{i}^{\prime}\right)\left(b_{i}-b_{i}^{\prime}\right)+l_{2}\left(b_{i}, b_{i}^{\prime}\right)\left(H\left(b_{i}\right)-H\left(b_{i}^{\prime}\right)\right),
\end{aligned}
$$

where

$$
\begin{aligned}
& l_{1}\left(b_{i}, b_{i}^{\prime}\right)=F\left(\phi\left(b_{i}\right)\right)^{n-1}-\frac{F\left(\phi\left(b_{i}\right)\right)^{n-1}-F\left(\phi\left(b_{i}^{\prime}\right)\right)^{n-1}}{b_{i}-b_{i}^{\prime}}\left(\phi\left(b_{i}\right)-b_{i}^{\prime}\right), \\
& l_{2}\left(b_{i}, b_{i}^{\prime}\right)=F\left(\phi\left(b_{i}^{\prime}\right)\right)^{n-1}\left(\phi\left(b_{i}\right)(1-\delta)+\delta \hat{P}_{\phi\left(b_{i}\right)}\left(\phi\left(b_{i}\right)\right)-b_{i}^{\prime}\right) .
\end{aligned}
$$

Consider any $b \in(0, \beta(1)]$ such that $\Xi(b)=0$. Then, (35) implies the existence of a neighborhood $\mathcal{N}(b)$ of $b$ such that

$$
\forall b_{i} \in \mathcal{N}(b), b_{i}^{\prime} \in \mathcal{N}(b), b_{i}^{\prime}<b_{i}: l_{1}\left(b_{i}, b_{i}^{\prime}\right) \leq 0
$$

Taken together, (33) and (36) imply for all $b_{i} \in \mathcal{N}(b)$ that $F(\phi)^{n-1}$ is differentiable at $b_{i}$ and

$$
\lim _{b_{i}^{\prime} \rightarrow b_{i}} \frac{F\left(\phi\left(b_{i}\right)\right)^{n-1}-F\left(\phi\left(b_{i}^{\prime}\right)\right)^{n-1}}{b_{i}-b_{i}^{\prime}}=\frac{F\left(\phi\left(b_{i}\right)\right)^{n-1}}{\phi\left(b_{i}\right)-b_{i}} .
$$


Hence, $\phi$ is differentiable at $b_{i} \in(b)$, and,

$$
\forall b, \Xi(b)=0, b_{i} \in \mathcal{N}(b): \phi^{\prime}\left(b_{i}\right)=\frac{1}{N\left(\phi\left(b_{i}\right), b_{i}\right)}
$$

Note also,

$$
\begin{aligned}
& \forall b, \delta M(\phi(b))=b, \phi \text { differentiable at } b: \\
& \quad \delta M^{\prime}(\phi(b)) \phi^{\prime}(b)=\lim _{b_{i} \searrow b} \frac{\delta M\left(\phi\left(b_{i}\right)\right)-\delta M(\phi(b))}{b_{i}-b} \leq 1
\end{aligned}
$$

because $\delta M\left(\phi\left(b_{i}\right)\right) \leq b_{i}$ for all $b_{i}$, by Lemma 7 .

By (30),

$$
\forall b, \Xi(b)=1, \phi \text { differentiable at } b: \delta M^{\prime}(\phi(b)) \phi^{\prime}(b)=1 \text {. }
$$

In summary, for all $b \in(0, \beta(1)]$ such that $\phi$ is differentiable at $b$,

$$
\phi^{\prime}(b)= \begin{cases}\frac{1}{N(\phi(b), b)} & \text { if } \delta M(\phi(b))<b \\ \min \left\{\frac{1}{N(\phi(b), b)}, \frac{1}{\delta M^{\prime}(\phi(b))}\right\} & \text { if } \delta M(\phi(b))=b\end{cases}
$$

by (31), (34), (37), (38), and (39). Next we show that $\beta$ is Lipschitz continuous. By (33), $F(\phi)^{n-1}$ is locally Lipschitz in $(0,1]$, implying that $\phi$ is locally Lipschitz in $(0,1]$. Hence,

$$
\forall c, d \in(0, \beta(1)]: \phi(d)-\phi(c)=\int_{c}^{d} \phi^{\prime}(b) \mathrm{d} b,
$$

where $\phi^{\prime}$ is given almost everywhere by (40).

The mapping $b \mapsto N(\phi(b), b)$ is bounded above on $[0, \beta(1)]$ because $N(\phi(b), b) \leq(n-$ 1) $f(\phi(b)) \phi(b) / F(\phi(b)) \rightarrow n-1$ as $b \rightarrow 0$. Moreover, $M^{\prime}$ is bounded above on $[0,1]$ by Lemma 2. Therefore, $\phi^{\prime}$ is bounded below by a positive number. Hence, $\beta$ is Lipschitz on $[0,1]$ by (41).

Because $\beta$ is the inverse of $\phi,(40)$ implies (18).

As for uniqueness, let $\beta$ and $\gamma$ be two Lipschitz continuous functions that satisfy (18). By definition of $K$, if $\beta(\theta)>\gamma(\theta)$ then $K(\theta, \beta(\theta))<K(\theta, \gamma(\theta))$, for all $\theta \in(0,1)$. Therefore, $\beta \leq \gamma$. The same argument shows $\beta \geq \gamma$. 
Next we show that $H$ satisfies

$$
h(b) \equiv H^{\prime}(b)=H(b) L_{\phi}(b) \text { a.e. } b \in(0, \beta(1)] .
$$

Consider any $b \in(0, \beta(1)]$. If $\Xi[b]=0$ and $b=\delta M(\phi(b))$, then $h(b)=0$ and, by (37), $R_{\phi}(b)=0$. Hence, $h(b)=0=H(b) L_{\phi}(b)$, as was to be shown. If $\Xi[b]=0$ and $b>\delta M(\phi(b))$, then $h(b)=0=H(b) L_{\phi}(b)$ by definition of $L_{\phi}$. Finally suppose that $\Xi[b]=1$. The function $\hat{r}$ is continuous. Hence, using Lemma 1 , the function $b^{\prime} \mapsto \hat{P}_{\phi\left(b^{\prime}\right)}\left(\phi\left(b^{\prime}\right)\right)$ is continuous on $(0,1]$. Therefore,

$$
\lim _{b^{\prime} \backslash b} k_{2}\left(b, b^{\prime}\right)=S_{\phi}(b) F(\phi(b))=\lim _{b^{\prime \prime} \backslash b} l_{2}\left(b^{\prime \prime}, b\right) \quad \forall b \in(0, \beta(1)] .
$$

Because $\phi$ is differentiable almost everywhere,

$$
\lim _{b^{\prime}>b} k_{1}\left(b, b^{\prime}\right)=R_{\phi}(b) F(\phi(b))=\lim _{b^{\prime \prime} \backslash b} l_{1}\left(b^{\prime \prime}, b\right) \quad \text { a.e. } b \in(0, \beta(1)] .
$$

From (29), (35), (43), and (44) we get (42).

It is straightforward that (19) satisfies (42). It remains to be shown that (42) together with the boundary condition $H(\beta(1))=1$ (which holds because the speculator does not bid more than necessary to win for sure) has only one solution. Observe that, for all $\epsilon>0, L$ is bounded above on $[\epsilon, \beta(1)]$ due to (9). Therefore, applying the Picard-Lindelöf Theorem to the differential equation (42) implies that $H$ is unique on $[\epsilon, 1]$. As a distribution function, $H$ is right-continuous at 0 and thus uniquely determined at 0 as well.

$Q E D$

We now turn to the equilibrium existence proof. Lemma 12 shows the existence of a solution for a class of discontinuous differential equations. These equations are constructed by altering a continuous differential equation such that if the solution function hits the boundary value 0 , the solution remains there until the slope becomes positive again.

To prove the existence of a solution, the differential equation is transformed into an upper hemi-continuous differential inclusion that allows a set of slopes (including 0) for the solution function if it reaches the value 0 , and allows only the slope 0 if a negative value is reached. For the differential inclusion a solution exists by a theorem of Aubin and Cellina 
(1984). One then shows that the solution in fact does not take negative values and solves the original differential equation almost everywhere.

Lemma 12 Let $\underline{\theta}, \bar{\theta} \in \mathbb{R}$ and consider a bounded and continuous function $\hat{N}:[\underline{\theta}, \bar{\theta}] \times \mathbb{R} \rightarrow \mathbb{R}$. Define

$$
\hat{K}(\theta, b)= \begin{cases}\hat{N}(\theta, b) & \text { if } b>0 \\ \max \{0, \hat{N}(\theta, b)\} & \text { if } b \leq 0\end{cases}
$$

Then the initial value problem

$$
\hat{\beta}(\underline{\theta})=0, \quad \hat{\beta}^{\prime}(\theta)=\hat{K}(\theta, \hat{\beta}(\theta)) \text { a.e. } \theta \in[\underline{\theta}, \bar{\theta}],
$$

has a Lipschitz continuous solution $\hat{\beta}$.

For all $\theta$ such that $\hat{\beta}(\theta)>0$, the function $\hat{\beta}$ is differentiable at $\theta$.

Proof. Following Aubin-Cellina (1984, p. 101), define

$$
\bar{K}(\theta, b)=\bigcap_{\epsilon>0} \overline{\mathrm{co}} \hat{K}\left(B_{\epsilon}(\theta, b)\right)
$$

where $B_{\epsilon}(\theta, b)$ denotes the $\epsilon$-ball around $(\theta, b)$ according to any norm in $\mathbb{R}^{2}$, and $\overline{c o}$ denotes the closed-convex-hull operator. Then, $\bar{K}$ is an upper hemi-continuous (or, in Aubin-Cellina's (1984) terminology, upper semi-continuous) correspondence, and its values are closed and convex. Moreover, $\bar{K}$ is globally bounded. Therefore, the differential inclusion problem

$$
\hat{\beta}^{\prime}(\theta) \in \bar{K}(\theta, \hat{\beta}(\theta)) \text { a.e. } \theta \in(\underline{\theta}, \bar{\theta}), \quad \hat{\beta}(\underline{\theta})=0,
$$

has an absolutely continuous solution $\hat{\beta}$ (see Aubin-Cellina, 1984, Theorem 4, p. 101). Note that, by (47), for a.e. $\theta \in[\underline{\theta}, \bar{\theta}]$ : if $\hat{\beta}(\theta)<0$ then $\hat{\beta}^{\prime}(\theta) \geq 0$. Hence,

$$
\forall \theta \in[\underline{\theta}, \bar{\theta}]: \hat{\beta}(\theta) \geq 0 .
$$

We will now show that $\hat{\beta}$ satisfies (46). First, consider $\theta \in[\underline{\theta}, \bar{\theta}]$ with $\hat{\beta}(\theta)>0$, or $\hat{\beta}(\theta)=0$ and $\hat{N}(\theta, 0) \geq 0$. Then, $\bar{K}(\theta, \hat{\beta}(\theta)=\hat{K}(\theta, \hat{\beta}(\theta))$.

Second, consider $\theta \in[\underline{\theta}, \bar{\theta}]$ with $\hat{\beta}(\theta)=0$ and $\hat{N}(\theta, 0)<0$. For a.e. such $\theta$, (47) implies $\hat{\beta}^{\prime}(\theta) \leq 0$. On the other hand, $\hat{\beta}^{\prime}(\theta) \geq 0$ by $(48)$. Therefore, $\hat{\beta}^{\prime}(\theta)=0=\hat{K}(\theta, \hat{\beta}(\theta))$, completing the proof of $(46)$. 
Because $\hat{\beta}$ is absolutely continuous,

$$
\forall \theta \in[\underline{\theta}, \bar{\theta}]: \hat{\beta}(\theta)=\int_{\underline{\theta}}^{\theta} \hat{K}\left(\theta^{\prime}, \hat{\beta}\left(\theta^{\prime}\right)\right) \mathrm{d} \theta^{\prime} .
$$

This together with the fact that $\hat{K}$ is bounded, implies that $\hat{\beta}$ is Lipschitz continuous. If $\hat{\beta}(\theta)>0$ for some $\theta$, then $\hat{K}\left(\theta^{\prime}, \hat{\beta}\left(\theta^{\prime}\right)\right)=\hat{N}\left(\theta^{\prime}, \hat{\beta}\left(\theta^{\prime}\right)\right)$ for all $\theta^{\prime}$ that are sufficiently close to $\theta$. Continuity of $\hat{N}$ together with (49) then implies that $\hat{\beta}$ is differentiable at $\theta$.

Let $\beta^{\text {I }}$ denote the standard equilibrium bid function of the first-price auction with $n$ regular bidders and no resale opportunity. The following result is well known.

LEMma 13 Let $N$ be defined as in (15). Then

$$
\beta^{I}(0)=0, \quad \forall \theta \in[0,1]: \beta^{I^{\prime}}(\theta)=N\left(\theta, \beta^{I}(\theta)\right)
$$

The next lemma constructs the equilibrium bid function $\beta$ for the regular bidders. One first shows that bidder types close to 0 make the same bid as in the absence of a resale opportunity. To extend the bid function to the higher types, Lemma 12 is applied to a differential equation for the excess of the regular bidders' bid function $\beta$ over the fictitious bid function $\delta M$ that corresponds to a 0 payoff for the speculator.

LEMMA 14 The initial value problem (18) has a solution $\beta$ on $[0,1]$ with the following properties:

$$
\begin{aligned}
& \beta \text { is Lipschitz continuous on }[0,1] \text {, } \\
& \forall \theta \in(0,1]: \beta(\theta)<\theta \text {, } \\
& \beta \text { is strictly increasing on }[0,1] \text {, } \\
& \forall \theta \in[0,1]: \beta(\theta) \geq \delta M(\theta) \text {, } \\
& \phi \equiv \beta^{-1} \text { is Lipschitz continuous on }[0, \beta(1)] .
\end{aligned}
$$


Proof. Define $\underline{\theta}$ to be the smallest $\theta \in[0,1]$ with $\beta^{\mathrm{I}}(\theta)=\delta M(\theta)$ (let $\underline{\theta}=1$ if no such $\theta$ exists). Because $\beta^{I^{\prime}}(0)=(n-1) / n>\delta M^{\prime}(0)$ by $(7)$, we have $\underline{\theta}>0$. Defining $\beta(\theta)=\beta^{\mathrm{I}}(\theta)$ for $\theta \in[0, \underline{\theta}]$, it follows from Lemma 13 that (18) is satisfied for $\theta \in[0, \underline{\theta}]$, and it follows from the theory of first-price auctions without resale that $\beta$ has the desired properties (50) to $(54)$ on $[0, \underline{\theta}]$.

Define $\hat{N}:[\underline{\theta}, 1] \times \mathbb{R} \rightarrow \mathbb{R}$ by

$$
\hat{N}(\theta, b)= \begin{cases}N(\theta, b+\delta M(\theta))-\delta M^{\prime}(\theta), & \text { if } b \in[0, \theta-\delta M(\theta)], \\ N(\theta, \theta)-\delta M^{\prime}(\theta), & \text { if } b>\theta-\delta M(\theta), \\ N(\theta, \delta M(\theta))-\delta M^{\prime}(\theta), & \text { if } b<0,\end{cases}
$$

and define $\hat{K}$ as in (45) with $\bar{\theta}=1$. Then, Lemma 12 implies that there exists a Lipschitz continuous $\hat{\beta}$ such that (46) holds. By definition of $\hat{K}$,

$$
\forall \theta \in[\underline{\theta}, 1]: 0 \leq \hat{\beta}(\theta) \leq \theta-\delta M(\theta) .
$$

Therefore, $\beta(\theta) \equiv \hat{\beta}(\theta)+\delta M(\theta), \theta \in[\underline{\theta}, 1]$, yields a Lipschitz continuous solution for (18).

To prove (51), suppose that $\beta(\theta) \geq \theta$ for some $\theta>\underline{\theta}$. Let $\theta^{\prime}$ be minimal with that property. Then, $\beta\left(\theta^{\prime}\right)=\theta^{\prime}>\delta M\left(\theta^{\prime}\right)$. Thus, $\beta$ is differentiable at $\theta^{\prime}$ by Lemma 12. Also, $\hat{N}(\theta, \hat{\beta}(\theta))=-\delta M^{\prime}(\theta)$. Hence, $\beta^{\prime}\left(\theta^{\prime}\right)=0$. Thus, $\beta(\theta)>\theta$ for some $\theta<\theta^{\prime}$, a contradiction.

To prove (52), observe that

$$
\beta^{\prime}(\theta) \stackrel{(18)}{=} K(\theta, \beta(\theta)) \stackrel{(14)}{\geq} N(\theta, \beta(\theta)) \stackrel{(51)}{>} 0 \text { a.e. } \theta \geq \underline{\theta} .
$$

This also implies that $\beta^{\prime}$ is bounded below by a positive number on $[\underline{\theta}, 1]$. Hence, $\phi$ is Lipschitz on $[\beta(\underline{\theta}), \beta(1)]$; i.e., (54) follows. Inequality (53) is immediate from (14) and (18). $Q E D$

The final lemma towards the proof of Proposition 1 shows equilibrium existence. We begin by showing that (19) yields a well-defined distribution function. Next, although by construction of the differential equation (18) the bid function $\beta$ satisfies the first-order condition for optimal bidding for every regular-bidder type, it remains to be shown is that 
$\beta$ is globally optimal. To get this, we show quasi-concavity of the regular bidders' payoff function. When considering upward deviations, we must take care of the possibility that a regular bidder deviates to a higher bid in an attempt to offer the good for resale. No such complication arises in the context of downward deviations because such a deviation does not lead to additional resale offers from competing regular bidders. Optimality of the speculator's bid distribution follows by construction.

LEMMA 15 The first-price auction with resale has a quasi-symmetric regular equilibrium $(\beta, H, \mathcal{M})$.

Proof. Define $\beta$ according to Lemma 14, and define $\phi=\beta^{-1}$. The first step is to show that $H$ as defined by (19) is a well-defined distribution function. For any given $\epsilon>0,(9)$ implies that there exists $\xi>0$ such that

$$
\forall b \in[\epsilon, \beta(1)]: \quad \text { if } b=\delta M(\phi(b)) \text { then } \delta \hat{P}_{\phi(b)}(\phi(b)) \geq b+\delta \xi
$$

By definition of $K$,

$$
\beta^{\prime}(\phi(b)) \geq N(\phi(b), \beta(\phi(b))) \text { a.e. } b \in(0, \beta(1)]
$$

implying

$$
R_{\phi}(b) \stackrel{(17)}{\geq} 0 \text { a.e. } b \in(0, \beta(1)]
$$

From (55) and (56) it follows that $L_{\phi}(\mathrm{cf}$. (16)) is well-defined almost everywhere, and is bounded above on $[\epsilon, \beta(1)]$ for any given $\epsilon>0$. Hence, $H$ is Lipschitz continuous on $[\epsilon, \beta(1)]$ for every $\epsilon>0$. Moreover, because $L_{\phi}$ is non-negative $H$ is weakly increasing on $(0, \beta(1)]$ and the limit $H(0)=\lim _{b \backslash 0} H(b)$ exists. Therefore, $H$ is a distribution function.

Let post-auction beliefs and resale mechanisms be defined by (10) and (11). It remains to be shown that (12) and (13) hold.

To show (12), first consider any deviating bid $b_{i} \in\left(0, \beta\left(\theta_{i}\right)\right)$ of a bidder $i \neq s$ with type $\theta_{i} \in(0,1]$. After winning at $b_{i}$, it is optimal for bidder $i$ to consume the good because she believes that the highest use value among bidders other than herself is at most $\phi\left(b_{i}\right)<\theta_{i}$. 
Also, if some bidder $j \in I \backslash\{i\}$ wins after making her equilibrium bid then bidder $j$ consumes the good in period 1 . Hence, bidder $i$ 's payoff is given by

$$
u_{i}\left(b_{i}, \theta_{i}\right)=H\left(b_{i}\right) F\left(\phi\left(b_{i}\right)\right)^{n-1}\left(\theta_{i}-b_{i}\right)+\delta \int_{b_{i}}^{\infty} F\left(\phi\left(b_{s}\right)\right)^{n-1} \hat{U}_{\phi\left(b_{s}\right)}\left(\theta_{i}\right) \mathrm{d} H\left(b_{s}\right),
$$

where

$$
\hat{U}_{\phi\left(b_{s}\right)}\left(\theta_{i}\right) \equiv \theta_{i} \hat{Q}_{\phi\left(b_{s}\right)}\left(\theta_{i}\right)-\hat{P}_{\phi\left(b_{s}\right)}\left(\theta_{i}\right)
$$

Because $\phi$ and $H$ are locally Lipschitz continuous on $(0, \beta(1)]$, the mapping $b_{i} \mapsto u_{i}\left(b_{i}, \theta_{i}\right)$ has the same property and is differentiable Lebesgue-a.e. in $(0,1)$. Hence, for a.e. $b_{i} \in\left(0, \beta\left(\theta_{i}\right)\right)$,

$$
\begin{array}{cl}
\frac{\partial u_{i}}{\partial b_{i}}\left(b_{i}, \theta_{i}\right)= & H\left(b_{i}\right) F\left(\phi\left(b_{i}\right)\right)^{n-2}\left((n-1) f\left(\phi\left(b_{i}\right)\right) \phi^{\prime}\left(b_{i}\right)\left(\theta_{i}-b_{i}\right)-F\left(\phi\left(b_{i}\right)\right)\right) \\
& +H^{\prime}\left(b_{i}\right) F\left(\phi\left(b_{i}\right)\right)^{n-1}\left(\theta_{i}-b_{i}-\delta \hat{U}_{\phi\left(b_{i}\right)}\left(\theta_{i}\right)\right) \\
& \theta_{i} \geq \phi\left(b_{i}\right) \\
& H\left(b_{i}\right) F\left(\phi\left(b_{i}\right)\right)^{n-1}\left((n-1) \frac{f\left(\phi\left(b_{i}\right)\right)}{F\left(\phi\left(b_{i}\right)\right)}\left(\phi\left(b_{i}\right)-b_{i}\right) \phi^{\prime}\left(b_{i}\right)-1\right) \\
& +H^{\prime}\left(b_{i}\right) F\left(\phi\left(b_{i}\right)\right)^{n-1}\left(\theta_{i}-b_{i}-\delta \hat{U}_{\phi\left(b_{i}\right)}\left(\theta_{i}\right)\right) .
\end{array}
$$

By definition of $R_{\phi}$ and because $H^{\prime}\left(b_{i}\right)=L_{\phi}\left(b_{i}\right) H\left(b_{i}\right)$ by $(19)$,

$$
\frac{\frac{\partial u_{i}}{\partial b_{i}}\left(b_{i}, \theta_{i}\right)}{H\left(b_{i}\right) F\left(\phi\left(b_{i}\right)\right)^{n-1}} \geq-R_{\phi}\left(b_{i}\right)+L_{\phi}\left(b_{i}\right)\left(\theta_{i}-b_{i}-\delta \hat{U}_{\phi\left(b_{i}\right)}\left(\theta_{i}\right)\right) .
$$

To show that

$$
\frac{\partial u_{i}}{\partial b_{i}}\left(b_{i}, \theta_{i}\right) \geq 0 \quad \text { a.e. } \quad b_{i} \in\left(0, \beta\left(\theta_{i}\right)\right),
$$

we distinguish two cases. If $b_{i}>\delta M\left(\phi\left(b_{i}\right)\right)$, then $\beta\left(\phi\left(b_{i}\right)\right)>\delta M\left(\phi\left(b_{i}\right)\right)$, hence $\beta^{\prime}\left(\phi\left(b_{i}\right)\right)=$ $N\left(\phi\left(b_{i}\right), b_{i}\right)$ by $(18)$. Thus, $R_{\phi}\left(b_{i}\right)=0$. Moreover, $L_{\phi}\left(b_{i}\right)=0$ by definition of $L_{\phi}$. Hence, (58) follows from (57).

If $b_{i}=\delta M\left(\phi\left(b_{i}\right)\right)$ then $R_{\phi}\left(b_{i}\right)=L_{\phi}\left(b_{i}\right) S_{\phi}\left(b_{i}\right)$ by definition of $L_{\phi}$. Using this and the definition of $S_{\phi}\left(b_{i}\right)$, (57) implies

$$
\frac{\frac{\partial u_{i}}{\partial b_{i}}\left(b_{i}, \theta_{i}\right)}{H\left(b_{i}\right) F\left(\phi\left(b_{i}\right)\right)^{n-1}} \geq L_{\phi}\left(b_{i}\right)\left(\theta_{i}-\delta \hat{U}_{\phi\left(b_{i}\right)}\left(\theta_{i}\right)-(1-\delta) \phi\left(b_{i}\right)-\delta \hat{P}_{\phi\left(b_{i}\right)}\left(\phi\left(b_{i}\right)\right)\right) \text {. }
$$

Now (58) follows from $\hat{U}_{\phi\left(b_{i}\right)}\left(\theta_{i}\right)=\theta_{i}-\hat{P}_{\phi\left(b_{i}\right)}\left(\phi\left(b_{i}\right)\right)$ by $(4)$.

From (58) it follows that type $\theta_{i}$ cannot gain from deviating to any bid $b_{i} \in\left[0, \beta\left(\theta_{i}\right)\right)$. 
Now consider a deviating bid $b_{i} \in\left(\beta\left(\theta_{i}\right), \beta(1)\right]$. Suppose first that bidder $i$ offers the good for resale upon winning. We obtain an upper bound $v_{i}\left(b_{i}, \theta_{i}\right)$ for bidder $i$ 's payoff by assuming she gets the entire surplus that is available in the resale market,

$$
\begin{aligned}
v_{i}\left(b_{i}, \theta_{i}\right)= & H\left(b_{i}\right)\left(F\left(\phi\left(b_{i}\right)\right)^{n-1}\left(\delta \theta_{i}-b_{i}\right)+\delta \int_{\theta_{i}}^{\phi\left(b_{i}\right)}\left(\theta^{\prime}-\theta_{i}\right) \mathrm{d} F\left(\theta^{\prime}\right)^{n-1}\right) \\
& +\delta \int_{b_{i}}^{\infty} F\left(\phi\left(b_{s}\right)\right)^{n-1} U_{\phi\left(b_{s}\right)}\left(\theta_{i}\right) \mathrm{d} H\left(b_{s}\right) .
\end{aligned}
$$

For a.e. $b_{i} \in\left(\beta\left(\theta_{i}\right), \beta(1)\right)$,

$$
\begin{aligned}
& \frac{\partial v_{i}}{\partial b_{i}}\left(b_{i}, \theta_{i}\right) \\
& \stackrel{\theta_{i} \leq \phi\left(b_{i}\right)}{\leq} H\left(b_{i}\right) F\left(\phi\left(b_{i}\right)\right)^{n-1}\left((n-1) \frac{f\left(\phi\left(b_{i}\right)\right)}{F\left(\phi\left(b_{i}\right)\right)}\left(\delta \phi\left(b_{i}\right)-b_{i}\right) \phi^{\prime}\left(b_{i}\right)-1\right) \\
& +H^{\prime}\left(b_{i}\right) F\left(\phi\left(b_{i}\right)\right)^{n-1}\left(\delta \theta_{i}-b_{i}-\delta \hat{U}_{\phi\left(b_{i}\right)}\left(\theta_{i}\right)+\delta \int_{\theta_{i}}^{\phi\left(b_{i}\right)} \frac{\theta^{\prime}-\theta_{i}}{F\left(\phi\left(b_{i}\right)\right)^{n-1}} \mathrm{~d}\left(F\left(\theta^{\prime}\right)^{n-1}\right)\right) .
\end{aligned}
$$

By definition of $R_{\phi}$ and $\delta \leq 1$, and because $H^{\prime}\left(b_{i}\right)=L_{\phi}\left(b_{i}\right) H\left(b_{i}\right)$ by (19),

$$
\begin{array}{r}
\frac{\frac{\partial v_{i}}{\partial b_{i}}\left(b_{i}, \theta_{i}\right)}{H\left(b_{i}\right) F\left(\phi\left(b_{i}\right)\right)^{n-1} \leq-} R_{\phi}\left(b_{i}\right)+L_{\phi}\left(b_{i}\right)\left(\delta \theta_{i}-b_{i}-\delta \hat{U}_{\phi\left(b_{i}\right)}\left(\theta_{i}\right)\right. \\
\left.+\delta \int_{\theta_{i}}^{\phi\left(b_{i}\right)} \frac{\theta^{\prime}-\theta_{i}}{F\left(\phi\left(b_{i}\right)\right)^{n-1}} \mathrm{~d} F\left(\theta^{\prime}\right)^{n-1}\right) .
\end{array}
$$

To show that

$$
\frac{\partial v_{i}}{\partial b_{i}}\left(b_{i}, \theta_{i}\right) \leq 0 \quad \text { a.e. } \quad b_{i} \in\left(\beta\left(\theta_{i}\right), \beta(1)\right),
$$

we distinguish two cases. If $b_{i}>\delta M\left(\phi\left(b_{i}\right)\right)$, then $R_{\phi}\left(b_{i}\right)=0$ by $(18)$ and $L_{\phi}\left(b_{i}\right)=0$ by definition of $L_{\phi}$. Hence, (60) follows from (59).

If $b_{i}=\delta M\left(\phi\left(b_{i}\right)\right)$ then $R_{\phi}\left(b_{i}\right)=L_{\phi}\left(b_{i}\right) S_{\phi}\left(b_{i}\right)$. Using this and the definition of $S_{\phi}\left(b_{i}\right)$, (59) implies

$$
\begin{aligned}
\frac{\frac{\partial v_{i}}{\partial b_{i}}\left(b_{i}, \theta_{i}\right)}{H\left(b_{i}\right) F\left(\phi\left(b_{i}\right)\right)^{n-1} \leq L_{\phi}\left(b_{i}\right)} & \left(\delta \theta_{i}-\delta \hat{U}_{\phi\left(b_{i}\right)}\left(\theta_{i}\right)+\delta \int_{\theta_{i}}^{\phi\left(b_{i}\right)} \frac{\theta^{\prime}-\theta_{i}}{F\left(\phi\left(b_{i}\right)\right)^{n-1}} \mathrm{~d}\left(F\left(\theta^{\prime}\right)^{n-1}\right)\right. \\
& \left.-(1-\delta) \phi\left(b_{i}\right)-\delta \hat{P}_{\phi\left(b_{i}\right)}\left(\phi\left(b_{i}\right)\right)\right) .
\end{aligned}
$$


Using the envelope theorem in integral form and integration by parts, the expression in brackets on the right-hand side of (61) can be simplified:

$$
\begin{aligned}
(\ldots)= & \delta \theta_{i}-\phi\left(b_{i}\right)+\delta\left(\hat{U}_{\phi\left(b_{i}\right)}\left(\phi\left(b_{i}\right)\right)-\hat{U}_{\phi\left(b_{i}\right)}\left(\theta_{i}\right)\right) \\
& +\delta \int_{\theta_{i}}^{\phi\left(b_{i}\right)} \frac{\theta^{\prime}-\theta_{i}}{F\left(\phi\left(b_{i}\right)\right)^{n-1}} \mathrm{~d}\left(F\left(\theta^{\prime}\right)^{n-1}\right) \\
= & \delta \theta_{i}-\phi\left(b_{i}\right)+\delta \int_{\theta_{i}}^{\phi\left(b_{i}\right)} \frac{F\left(\theta^{\prime}\right)^{n-1}}{F\left(\phi\left(b_{i}\right)\right)^{n-1}} \mathrm{~d} \theta^{\prime}+\delta \int_{\theta_{i}}^{\phi\left(b_{i}\right)} \frac{\theta^{\prime}-\theta_{i}}{F\left(\phi\left(b_{i}\right)\right)^{n-1}} \mathrm{~d}\left(F\left(\theta^{\prime}\right)^{n-1}\right) \\
= & \delta \theta_{i}-\phi\left(b_{i}\right)+\left.\delta \theta^{\prime} \frac{F\left(\theta^{\prime}\right)^{n-1}}{F\left(\phi\left(b_{i}\right)\right)^{n-1}}\right|_{\theta_{i}} ^{\phi\left(b_{i}\right)}-\delta \theta_{i} \frac{F\left(\phi\left(b_{i}\right)\right)^{n-1}-F\left(\theta_{i}\right)^{n-1}}{F\left(\phi\left(b_{i}\right)\right)^{n-1}} \\
= & -(1-\delta) \phi\left(b_{i}\right) \leq 0 .
\end{aligned}
$$

Hence, (60) follows from (61).

Finally, consider a deviating bid $b_{i} \in\left(\beta\left(\theta_{i}\right), \beta(1)\right]$ and suppose that bidder $i$ consumes the good upon winning. Let $\check{v}_{i}\left(b_{i}, \theta_{i}\right)$ denote the corresponding payoff. Using techniques analogous to those leading to (58) and (60), one shows that

$$
\frac{\partial \check{v}_{i}}{\partial b_{i}}\left(b_{i}, \theta_{i}\right) \leq 0 \quad \text { a.e. } \quad b_{i} \in\left(\beta\left(\theta_{i}\right), \beta(1)\right) .
$$

An upper bound for bidder $i$ 's payoff with any bid $b_{i} \in\left(\beta\left(\theta_{i}\right), \beta(1)\right]$ is

$$
\bar{u}_{i}\left(b_{i}, \theta_{i}\right) \equiv \max \left\{v_{i}\left(b_{i}, \theta_{i}\right), \check{v}_{i}\left(b_{i}, \theta_{i}\right)\right\}
$$

Because the maximum of two locally Lipschitz continuous functions is locally Lipschitz itself, the mapping $b_{i} \mapsto \bar{u}_{i}\left(b_{i}, \theta_{i}\right)$ can be written as the integral over its derivative. Moreover, $\bar{u}_{i}\left(\beta\left(\theta_{i}\right), \theta_{i}\right)=u_{i}\left(\beta\left(\theta_{i}\right), \theta_{i}\right)$. Therefore, (60) and (62) imply that for all $b_{i} \in\left(\beta\left(\theta_{i}\right), \beta(1)\right]$,

$$
\begin{aligned}
u_{i}\left(b_{i}, \theta_{i}\right)-u_{i}\left(\beta\left(\theta_{i}\right), \theta_{i}\right) \leq & \bar{u}_{i}\left(b_{i}, \theta_{i}\right)-\bar{u}_{i}\left(\beta\left(\theta_{i}\right), \theta_{i}\right) \\
& =\int_{\beta\left(\theta_{i}\right)}^{b_{i}} \frac{\partial \bar{u}_{i}}{\partial b_{i}}\left(b, \theta_{i}\right) \mathrm{d} b \leq 0 .
\end{aligned}
$$

Hence, no type $\theta_{i}>0$ has an incentive to deviate. By continuity of $u_{i}$, type $\theta_{i}=0$ has no incentive to deviate either. This completes the proof of (12).

To complete the equilibrium existence proof, we have to show (13). From (53) it follows that $b_{s} \geq \delta M\left(\phi\left(b_{s}\right)\right)$, and thus $u_{s}\left(b_{s}\right) \leq 0$, for all $b_{s} \in(0, \beta(1)]$. It remains to be shown that

$$
\operatorname{Pr}\left[u_{s}\left(\tilde{b}_{s}\right)<0\right]=0
$$


Consider the event $u_{s}\left(\tilde{b}_{s}\right)<0$. Then, $\beta\left(\phi\left(\tilde{b}_{s}\right)\right)>\delta M\left(\phi\left(\tilde{b}_{s}\right)\right)$ and thus $L_{\phi}\left(\tilde{b}_{s}\right)=0$. Using (19), the probability of that event is

$$
\int_{(0, \beta(1)]} \mathbf{1}_{L_{\phi}\left(b_{s}\right)=0} h\left(b_{s}\right) \mathrm{d} b_{s}=\int_{(0, \beta(1)]} \mathbf{1}_{L_{\phi}\left(b_{s}\right)=0} L_{\phi}\left(b_{s}\right) H\left(b_{s}\right) \mathrm{d} b_{s}=0 .
$$

$Q E D$

This completes the proof of Proposition 1.

Observe that Proposition 1 allows the possibility that $H(0)=1$; i.e., the speculator may not play an active role at all. The following Proposition 2 shows that the answer to the question whether the speculator plays an active role depends on the distribution $F$, the discount factor $\delta$, and the number of regular bidders $n$. For any given $n$, Proposition 2 determines the smallest discount factor $\delta_{n}$ such that the speculator plays an active role for some $F$. To this end, we show that the type of distribution described on p. 18 of the Note is the most favorable for speculative activity so that we can define

$$
\delta_{n}=\min _{z \in[0,1]} \frac{\eta(z, n)}{\rho(z, n)} .
$$

The intuition is as follows. Because the distribution of the types below the resale reserve price is irrelevant for the resale revenue, the most favorable distribution for the speculator is the one with the smallest expectation conditional on the information that the value is below the reserve price. One cannot shift all the probability weight to 0 because that would violate the increasing hazard rate assumption; the best one can do is to shift probability weight to lower values until the hazard rate is constant - this yields the exponential shape. Above the resale reserve price the best that can happen for the speculator is that all the probability weight is concentrated just above the reserve price so that the bidders obtain no information rent. This yields the type of distribution of the above example.

Proposition 2 For every regular-bidder number $n \geq 2$, there exists a discount factor $\delta_{n}<1$ such that the following holds in the quasi-symmetric regular equilibrium of a first-price or Dutch auction with resale: 
(i) for all $\delta>\delta_{n}$, there exists a distribution $F$ satisfying Assumption 1 such that the speculator plays an active role;

(ii) for all $\delta \leq \delta_{n}$ and all $F$ satisfying Assumption 1, the speculator bids 0 and the regular bidders use the same bid function as in the absence of a resale opportunity.

Moreover, $\delta_{n} \rightarrow 1$ as $n \rightarrow \infty$.

Proof. Observe that, for all $(F, n, \delta)$,

$$
\text { if } H(0)=1 \text { then } \beta=\beta^{\text {I }} \text {. }
$$

To see $(64)$, suppose that $H(0)=1$. Then (19) implies $L_{\phi}(b)=0$ a.e. $b \in[0, \beta(1)]$. Hence, $\beta=\beta^{\mathrm{I}}$ by Lemma 13, (16), (17), and (18). ${ }^{9}$

The argument given in Section 5 of the Note together with (64) proves part (i). To prove the "Moreover" part, note that

$$
\rho(z, n) \leq 1, \quad \min _{z \in[0,1]} \eta(z, n) \rightarrow_{n \rightarrow \infty} 1 .
$$

To prove part (ii), we need additional notation concerning order statistics. For any distribution function $D$, let $D^{(k, l)}$ denote the distribution function for the lth-highest order statistic among $k$ i.i.d. random variables $(k=1,2 \ldots ; l=1, \ldots, k)$ that are distributed according to D.

Fix any $n \geq 2, \delta \leq \delta_{n}$, and $F$ with an increasing hazard rate. Suppose that $\beta=\beta^{\mathrm{I}}$. To complete the proof of (ii), it is sufficient to show that $u_{s}\left(b_{s}\right) \leq 0$ for all $b_{s} \geq 0$ (because then $H$ with $H(0)=1$ is a best-response bid distribution of the speculator, and $\beta$ is a best-response bid function of the regular bidders by (64)).

By definition of $\beta^{\mathrm{I}}$, if $b_{s}=\beta^{\mathrm{I}}(1)$,

$$
u_{s}\left(b_{s}\right)=\pi(F, n, \delta) \equiv \delta M(1)-\int_{0}^{1} \theta \mathrm{d} F^{(n-1,1)}(\theta)
$$

If $b_{s}<\beta^{\mathrm{I}}(1)$ and $\hat{\theta} \equiv\left(\beta^{\mathrm{I}}\right)^{-1}\left(b_{s}\right)$,

$$
u_{s}\left(b_{s}\right)=F(\hat{\theta})^{n}\left(\delta M(\hat{\theta})-\int_{0}^{1} \theta \mathrm{d} \hat{F}_{[0, \hat{\theta}]}^{(n-1,1)}(\theta)\right)=F(\hat{\theta})^{n} \pi\left(\hat{F}_{[0, \hat{\theta}]}, n, \delta\right) .
$$

\footnotetext{
${ }^{9}$ The result that the bid function $\beta=\beta^{\mathrm{I}}$ yields an equilibrium in the absence of bids by the speculator already appears in Haile (1999, Theorem 1).
} 
Hence, w.l.o.g. it is sufficient to show that

$$
\pi(F, n, \delta) \leq 0 .
$$

Rearranging (5), one finds

$$
M(1)=n(1-F(\check{r})) F(\check{r})^{n-1} \check{r}+\int_{\check{r}}^{1} \theta \mathrm{d} F^{(n, 2)}(\theta),
$$

where $\check{r} \equiv \hat{r}(1)$. Hence, using (65),

$$
\begin{aligned}
& \pi(F, n, \delta) \\
& =\delta\left(n(1-F(\check{r})) F(\check{r})^{n-1} \check{r}+\int_{\check{r}}^{1} \theta \mathrm{d} F^{(n, 2)}(\theta)\right)-\int_{0}^{1} \theta \mathrm{d} F^{(n-1,1)}(\theta) .
\end{aligned}
$$

Observe that $\check{r}=1 / \lambda(\check{r})$ by definition of the function $\hat{r}$, where $\lambda(\theta)=f(\theta) /(1-F(\theta))$ denotes for all $\theta \in[0,1)$ the hazard function for $F$. We can write

$$
F(\theta)=1-e^{-\int_{0}^{\theta} \lambda(t) \mathrm{d} t} .
$$

Let $\mu=(1 / \check{r}) \int_{0}^{\check{r}} \lambda(t) \mathrm{d} t$. Then

$$
\int_{0}^{\check{r}}(\lambda(t)-\mu) \mathrm{d} t=0 .
$$

Because $\lambda(t)-\mu$ is weakly increasing in $t$,

$$
\forall \theta \in[0, \check{r}]: \int_{0}^{\theta} \lambda(t) \mathrm{d} t \leq \int_{0}^{\theta} \mu \mathrm{d} t=\theta \mu .
$$

Therefore,

$$
\forall \theta \in[0, \check{r}]: \quad F(\theta) \leq J(\theta) \equiv 1-e^{-\theta \mu} .
$$

Also define $J(\theta)=F(\theta)$ for $\theta \in(\check{r}, 1]$. Then $J(\check{r})=F(\check{r})$ and $F$ stochastically dominates $J$. Therefore, using (67),

$$
\begin{aligned}
\pi(F, n, \delta) \leq \check{\pi} \equiv \delta( & \left.n(1-J(\check{r})) J(\check{r})^{n-1} \check{r}+\int_{\check{r}}^{1} \theta \mathrm{d} J^{(n, 2)}(\theta)\right) \\
& -\int_{0}^{1} \theta \mathrm{d} J^{(n-1,1)}(\theta) .
\end{aligned}
$$

For arbitrary i.i.d. random variables with densities, the highest of $n-1$ dominates the 2 nd highest of $n$ in terms of the likelihood ratio. Therefore, the expectation of the highest of $n-1$ 
conditional on being greater or equal to $\check{r}$ is greater or equal to the respective conditional expectation of the 2 nd highest of $n$,

$$
\frac{1}{1-J^{(n, 2)}(\check{r})} \int_{\check{r}}^{1} \theta \mathrm{d} J^{(n, 2)}(\theta) \leq \check{e} \equiv \frac{1}{1-J^{(n-1,1)}(\check{r})} \int_{\check{r}}^{1} \theta \mathrm{d} J^{(n-1,1)}(\theta) .
$$

Therefore, using the definition of $\check{\pi}$ from (68),

$$
\begin{aligned}
\check{\pi} \leq & \delta\left(n(1-J(\check{r})) J(\check{r})^{n-1} \check{r}+\left(1-J^{(n, 2)}(\check{r})\right) \check{e}\right) \\
& -\int_{0}^{\check{r}} \theta \mathrm{d} J^{(n-1,1)}(\theta)-\left(1-J^{(n-1,1)}(\check{r})\right) \check{e} .
\end{aligned}
$$

Because $J^{(n-1,1)}(\check{r}) \leq J^{(n, 2)}(\check{r})$ and $\check{e} \geq \check{r}$, it follows that

$$
\begin{aligned}
\check{\pi} \leq & \delta\left(n(1-J(\check{r})) J(\check{r})^{n-1} \check{r}+\left(1-J^{(n, 2)}(\check{r})\right) \check{r}\right) \\
& -\int_{0}^{\check{r}} \theta \mathrm{d} J^{(n-1,1)}(\theta)-\left(1-J^{(n-1,1)}(\check{r})\right) \check{r} \\
= & \delta\left(1-\left(1-e^{-z}\right)^{n}\right) \check{r} \\
& -\check{r}(n-1) \frac{1}{z} \int_{0}^{z} \tau e^{-\tau}\left(1-e^{-\tau}\right)^{n-2} \mathrm{~d} \tau-\left(1-\left(1-e^{-z}\right)^{n-1}\right) \check{r} \\
= & \check{r}(\delta \rho(z, n)-\eta(z, n))
\end{aligned}
$$

where $z \equiv \mu \check{r}=\mu / \lambda(\check{r}) \leq 1$ and we have made the substitution $\tau=\theta \mu$ in the integral. Because $\delta \leq \delta_{n}$, (63) implies $\delta \rho(z, n)-\eta(z, n) \leq 0$. Hence, (69) together with (68) shows (66).

Proposition 3 below shows that the presence of a resale opportunity never reduces initial seller revenue, and strictly increases it if the speculator plays an active role. This result follows because each regular-bidder type bids at least as much in the presence of a resale opportunity as without one, and the initial seller collects the speculator's bid if she wins. The proof below derives the result by comparing the differential equation (18) with the differential equation that is relevant in the absence of a resale opportunity (cf. Lemma 13). ${ }^{10}$

\footnotetext{
${ }^{10} \mathrm{~A}$ direct proof that does not go through the differential equation (18) would follow the arguments leading to (34) in the proof of Lemma 11, which is sufficient to make the appropriate comparison of differential equations.
} 
Proposition 3 For any regular-bidder number $n \geq 2$, discount factor $\delta \in(0,1)$, and distribution $F$ satisfying Assumption 1, the presence of a resale opportunity does not reduce initial seller revenue from a first-price or Dutch auction, and strictly increases it if $(F, n, \delta)$ is such that the speculator plays an active role.

ProOF. From (18) it follows

$$
\beta^{\prime}(\theta) \geq N(\theta, \beta(\theta)) \text { a.e. } \theta \in[0,1] .
$$

By $\beta(0)=0$, Lemma 13, and (70),

$$
\forall \theta \in[0,1]: \beta(\theta) \geq \beta^{\mathrm{I}}(\theta)
$$

This shows that the resale opportunity never reduces initial seller revenue. If the speculator wins with positive probability, the resale opportunity strictly increases initial seller revenue because the initial seller collects the speculator's bid.

$Q E D$

\section{The second-price auction with resale}

In this section we construct and discuss a continuum of pure-strategy perfect Bayesian equilibria for second-price auctions with resale where the speculator plays an active role. Proposition 4 describes the equilibria. Proposition 5 and Proposition 6 evaluate the impact of a resale opportunity on initial seller revenue.

Let $\beta$ denote bidder $i$ 's $(i \in I)$ bid in the second-price auction as a function of her use value. Let $\hat{b}_{s}=\tilde{b}_{s}$ denote the speculator's bid (we do not allow randomization, hence $\tilde{b}_{s}$ is a degenerate random variable).

To define post-auction beliefs, consider any bidder $j \in I$ and let $b_{j} \geq 0$ denote her bid. Let $i \in I \cup\{s\}$ denote the label of the winner. Then, the probability distribution $\Pi_{j}\left(\cdot \mid i, b_{j}\right)$ denotes the post-auction belief about $j$ 's use value of bidders other than $j$.

Let $\mathcal{M}\left(i, \mathbf{b}_{-i}, \theta_{i}\right)$ denote the resale mechanism used by the resale seller $i \in I \cup\{s\}$ after a second-price auction when the vector of losers' bids is $\mathbf{b}_{-i} \in[0, \infty)^{n}$ and $i$ 's use value is $\theta_{i}$. For all $i, j \in I \cup s$ with $j \neq i$, all $\mathbf{b}_{-i}$, and all $\theta_{i}, \theta_{j}$, let $P_{j}\left(i, \mathbf{b}_{-i}, \theta_{i}, \theta_{j}\right)$ denote the net 
expected transfer from bidder $j$ of type $\theta_{j}$ to the other bidders (including the transfer to i) in the mechanism $\mathcal{M}\left(i, \mathbf{b}_{-i}, \theta_{i}\right)$. Let $Q_{j}\left(i, \mathbf{b}_{-i}, \theta_{i}, \theta_{j}\right)$ denote the probability that bidder $j$ obtains the good. Let $P\left(i, \mathbf{b}_{-i}, \theta_{i}\right)$ denote the expected transfer to the resale seller $i$, and $Q\left(i, \mathbf{b}_{-i}, \theta_{i}\right)$ the probability that the resale seller keeps the good.

For all $i \in I$, bidder $i$ 's expected payoff when she bids $b_{i} \geq 0$ and has the use value $\theta_{i}$ equals

$$
\begin{aligned}
& u_{i}\left(b_{i}, \theta_{i}\right)=E[ \\
& \left(-\tilde{b}_{-i}^{(1)}+\max \left\{\theta_{i}, \delta\left(\theta_{i} Q\left(i, \tilde{\mathbf{b}}_{-i}, \theta_{i}\right)+P\left(i, \tilde{\mathbf{b}}_{-i}, \theta_{i}\right)\right)\right\}\right) \mathbf{1}_{w\left(b_{i}, \tilde{\mathbf{b}}_{-i}\right)=i} \\
& \left.+\sum_{j \neq i} \delta\left(\theta_{i} Q_{i}\left(j,\left(b_{i}, \tilde{\mathbf{b}}_{-j-i}\right), \tilde{\theta}_{j}, \theta_{i}\right)-P_{i}\left(j,\left(b_{i}, \tilde{\mathbf{b}}_{-j-i}\right), \tilde{\theta}_{j}, \theta_{i}\right)\right) \mathbf{1}_{w\left(b_{i}, \tilde{\mathbf{b}}_{-i}\right)=j}\right],
\end{aligned}
$$

where $w$ denotes the period- 1 winner as a function of the bid profile, and where the max-term reflects the condition that after winning in period 1 bidder $i$ decides optimally whether to consume the good or offer it for resale. The speculator's payoff when she bids $b_{s} \geq 0$ is given by

$$
u_{s}\left(b_{s}\right)=E\left[\left(-\tilde{b}_{-s}^{(1)}+\delta P\left(s, \tilde{\mathbf{b}}_{-s}, \theta_{s}\right)\right) \mathbf{1}_{w\left(b_{s}, \tilde{\mathbf{b}}_{-s}\right)=s}\right] .
$$

The equilibrium conditions are (71) that post-auction beliefs about auction losers are determined by Bayes rule whenever possible, (72) that the resale mechanism is chosen according to Assumption 4, and $(73,74)$ that period-1 behavior is optimal.

Definition 2 A tuple $\left(\beta, \hat{b}_{s}, \mathcal{M}\right)$ is a quasi-symmetric regular equilibrium of the secondprice auction with resale if there exists a belief system $\left(\Pi_{j}\left(\cdot \mid i, b_{j}\right)\right)_{j \in I, i \in I \cup\{s\}, b_{j} \geq 0}$ such that the following holds:

$$
\begin{aligned}
& \forall i \in I \cup\{s\}, j \in I \backslash\{i\}, b_{j} \geq 0: \\
& \Pi_{j}\left(\cdot \mid i, b_{j}\right)=\hat{F}_{\beta^{-1}\left(b_{j}\right)} \quad \text { if } b_{j} \in \beta([0,1]), \\
& \forall i \in I \cup\{s\}, \mathbf{b}_{-i} \in[0, \infty)^{n}, \theta_{i}: \\
& \mathcal{M}\left(i, \mathbf{b}_{-i}, \theta_{i}\right)=\hat{\mathcal{M}}\left(\left(\Pi_{j}\left(\cdot \mid i, b_{j}\right)\right)_{j \in I}, \theta_{i}, i\right),
\end{aligned}
$$




$$
\begin{gathered}
\forall i \neq s, \theta_{i}: \beta\left(\theta_{i}\right) \in \arg \max _{b_{i} \geq 0} u_{i}\left(b_{i}, \theta_{i}\right), \\
\hat{b}_{s} \in \arg \max _{b_{s} \geq 0} u_{s}\left(b_{s}\right) .
\end{gathered}
$$

This equilibrium concept is in the spirit of perfect Bayesian equilibrium, combined with the symmetry and regularity restrictions formulated in Assumption 2. Like in the first-price auction case (see the explanation below Definition 1), an equilibrium condition on the postauction beliefs about the auction winner is omitted because it would play no role for our analysis.

The result below describes our equilibria. The structure of these equilibria is analogous to the equilibria constructed in Proposition 2 of the Note. Condition (77) states that the resale mechanism used by the speculator is a standard auction with an optimal reserve price. Observe that if the speculator wins at a strictly positive price, then she is certain about the maximum use value among all bidders (see (78)), hence the standard auction yields the same outcome as a take-it-or-leave-it offer equal to the auction's reserve price.

Proposition 4 For any regular-bidder number $n \geq 2$, discount factor $\delta \in(0,1)$, and distribution F satisfying Assumption 1, and every $\theta^{*} \in[0,1]$, the second-price auction with resale has a quasi-symmetric regular equilibrium $\left(\beta, \hat{b}_{s}, \mathcal{M}\right)$ with the properties (75), (76), (77). It is supported by beliefs satisfying (78).

$$
\begin{gathered}
\forall \theta_{i}: \beta\left(\theta_{i}\right)=\left\{\begin{array}{c}
0 \quad \text { if } \theta_{i} \in\left[0, \theta^{*}\right), \\
\theta_{i} \quad \text { if } \theta_{i} \in\left(\theta^{*}, 1\right] .
\end{array}\right. \\
\hat{b}_{s}=\theta^{*}-\delta\left(\theta^{*}-\hat{P}_{\theta^{*}}\left(\theta^{*}\right)\right) . \\
\forall \mathbf{b}_{-s}: \mathcal{M}\left(s, \mathbf{b}_{-s}, 0\right)=\left\{\begin{array}{cc}
\mathcal{S}\left(\hat{r}\left(\theta^{*}\right)\right) & \text { if } b_{-s}^{(1)}=0, \\
\mathcal{S}\left(\theta^{*}\right) & \text { if } b_{-s}^{(1)} \in\left(0, \theta^{*}\right), \\
\mathcal{S}\left(b_{-s}^{(1)}\right) & \text { if } b_{-s}^{(1)} \in\left(\theta^{*}, 1\right] .
\end{array}\right. \\
\forall i, j \notin\{s, i\}, b_{j}, \theta_{j}: \Pi_{j}\left(\theta_{j} \mid i, b_{j}\right)=\left\{\begin{array}{cc}
\hat{F}_{\left[0, \theta^{*}\right]}\left(\theta_{j}\right) & \text { if } b_{j}=0, \\
\mathbf{1}_{\theta_{j} \geq \theta^{*}} & \text { if } b_{j} \in\left(0, \theta^{*}\right), \\
\mathbf{1}_{\theta_{j} \geq b_{j}} & \text { if } b_{j} \in\left(\theta^{*}, 1\right] .
\end{array}\right.
\end{gathered}
$$


The proof is a straightforward generalization of the arguments leading to the proof of Proposition 2 in the Note, except for the following complication. With multiple private-value bidders, we have to show that a regular bidder with type $\theta_{i}<\theta^{*}$ will not deviate to a bid in $\left[\hat{b}_{s}, \theta^{*}\right]$ and subsequently offer the good for resale if she wins. A detailed proof is below; here is a sketch of the argument. Suppose that bidder $i$ makes such a deviation and suppose if she wins then she appropriates the entire expected surplus that is available in the resale market. Because an increase of $\theta_{i}$ changes the resale surplus only if all bidders other than $i$ have types below $\theta_{i}$, the marginal change in expected resale surplus due to an increase of $\theta_{i}$ equals the probability that all bidders other than $i$ have types below $\theta_{i}$. But this probability equals (or is an upper bound for, if $\theta_{i}$ is below the resale reserve price $\left.\hat{r}\left(\theta^{*}\right)\right)$ the probability that bidder $i$ obtains the good if she bids 0 and waits for the speculator's resale mechanism. Hence, using the envelope theorem, the derivative of bidder $i$ 's equilibrium payoff with respect to $\theta_{i}$ is at most as great as the derivative of the expected resale surplus obtained from the deviation. Hence, if for any type $\theta_{i}<\theta^{*}$ the deviation it is profitable then it the deviation is also profitable for type $\theta_{i}=\theta^{*}$. But for type $\theta^{*}$ the deviation is not profitable by construction of $\hat{b}_{s}$.

Proof of Proposition 4. The proof of (71), (72), and (74), is straightforward. The proof of (73) is straightforward, too, except for one step: we have to show that a bidder $i \in I$ with type $\theta_{i} \leq \theta^{*}$ cannot profit from deviating to a bid $b_{i} \in\left[\hat{b}_{s}, \theta^{*}\right]$ and offering the good for resale if she wins. Observe that bidder $i$ 's resale payoff cannot exceed the entire expected surplus that is available in the resale market:

$$
u_{i}\left(b_{i}, \theta_{i}\right) \leq \bar{u}_{i}\left(\theta_{i}\right) \equiv\left(-\hat{b}_{s}+\delta E\left[\max \left\{\theta_{i}, \tilde{\theta}_{-i}^{(1)}\right\} \mid \tilde{\theta}_{-i}^{(1)} \leq \theta^{*}\right]\right) F\left(\theta^{*}\right)^{n-1} .
$$

A straightforward computation shows that

$$
\frac{\partial \bar{u}_{i}}{\partial \theta_{i}}\left(\theta_{i}\right)=\delta F\left(\theta_{i}\right)^{n-1}
$$

Bidder $i$ 's equilibrium payoff equals

$$
u_{i}\left(0, \theta_{i}\right)=\delta\left(\theta_{i} \hat{Q}_{\theta^{*}}\left(\theta_{i}\right)-\hat{P}_{\theta^{*}}\left(\theta_{i}\right)\right) F\left(\theta^{*}\right)^{n-1} .
$$


The Envelope Theorem implies

$$
\frac{\partial u_{i}}{\partial \theta_{i}}\left(0, \theta_{i}\right)= \begin{cases}\delta F\left(\theta_{i}\right)^{n-1} & \text { if } \theta_{i}>\hat{r}\left(\theta^{*}\right) \\ 0 & \text { if } \theta_{i}<\hat{r}\left(\theta^{*}\right)\end{cases}
$$

By (76),

$$
u_{i}\left(0, \theta^{*}\right) \geq \bar{u}_{i}\left(\theta^{*}\right)
$$

Hence, for all $\theta_{i}^{\prime} \leq \theta^{*}$,

$$
\begin{aligned}
u_{i}\left(0, \theta_{i}^{\prime}\right)-\bar{u}_{i}\left(\theta_{i}^{\prime}\right) & \stackrel{(82)}{\geq} u_{i}\left(0, \theta_{i}^{\prime}\right)-u_{i}\left(0, \theta^{*}\right)-\left(\bar{u}_{i}\left(\theta_{i}^{\prime}\right)-\bar{u}_{i}\left(\theta^{*}\right)\right) \\
& =\int_{\theta_{i}^{\prime}}^{\theta^{*}}\left(\frac{\partial \bar{u}_{i}}{\partial \theta_{i}}\left(\theta_{i}\right)-\frac{\partial u_{i}}{\partial \theta_{i}}\left(0, \theta_{i}\right)\right) \mathrm{d} \theta_{i} \\
\stackrel{(80),(81)}{=} & \geq 0,
\end{aligned}
$$

which completes the proof.

In contrast to the first-price/Dutch auction setting (cf. Proposition 3), the presence of a resale opportunity may either increase or decrease initial seller revenue, depending on which $\theta^{*}$-equilibrium is played. This comparison assumes that in the absence of a resale opportunity all bidders use the dominant strategy of bidding their use values.

Proposition 5 For any regular-bidder number $n \geq 2$, discount factor $\delta \in(0,1)$, and distribution $F$ satisfying Assumption 1, the second-price auction with resale has quasisymmetric regular equilibria such that the initial seller's expected revenue is larger (alternatively, smaller) than the expected revenue that results when all bidders bid their use values.

Here is a sketch of the proof. In the $\theta^{*}$-equilibrium with $\theta^{*}=0$ all bidders bid their use values. It is thus sufficient to show that the revenue of a $\theta^{*}$-equilibrium with $\theta^{*}>0$ is larger than the revenue of a 0 -equilibrium if $\theta^{*}$ is close to 0 , and smaller if $\theta^{*}$ is close to 1 . There exist two distinct events that cause the revenue of a $\theta^{*}$-equilibrium with $\theta^{*}>0$ to differ from the revenue that arises in the 0 -equilibrium. Event (i) is that $\theta^{*}$ lies between the highest and the second-highest use value, in which case revenue changes from the second-highest 
use value to the speculator's bid. Event (ii) is that $\theta^{*}$ is larger than the highest bidder use value, in which case revenue falls from the second-highest use value to 0 . If $\theta^{*}$ approaches 1, the probability of (i) becomes small and the probability of (ii) does not; this implies that expected revenue is reduced. If $\theta^{*}$ tends to 0 both events' probabilities tend to 0 , but event (i) allows one buyer's use value to stay above $\theta^{*}$ and thus becomes infinitely more likely than event (ii). The expected payoff loss from event (ii) is of the order $\theta^{*}$. It is thus sufficient to show that event (i) results in an expected payoff gain of the order $\theta^{*}$. Using the fact that the expected resale payment of type $\theta^{*}$ exceeds the speculator's bid, one obtains a lower bound for the expected payoff difference in terms of the underlying distribution $F$. Using properties of order statistics, one can verify that the payoff difference is positive of the order $\theta^{*}$ if $F$ is uniform. Because any continuously differentiable distribution is approximately uniform on any small interval, the argument extends to any $F$.

Proof of Proposition 5. For all $\theta^{*} \in[0,1]$, let $R\left(\theta^{*}\right)$ denote the initial seller's expected revenue in a $\theta^{*}$-equilibrium. In particular, $\pi(0)$ is the expected revenue of the initial seller when every bidder bids her use value in period 1 . We will show that $R\left(\theta^{*}\right)>R(0)$ for all $\theta^{*}$ sufficiently close to 0 and $R\left(\theta^{*}\right)<R(0)$ for all $\theta^{*}$ sufficiently close to 1 .

Let $\tilde{\theta}^{(1)}$ and $\tilde{\theta}^{(2)}$ denote the highest and second highest use value among the regular bidders.

$$
\begin{gathered}
R\left(\theta^{*}\right)-R(0)=\operatorname{Pr}\left[\tilde{\theta}^{(2)}<\theta^{*}<\tilde{\theta}^{(1)}\right]\left(\hat{b}_{s}-E\left[\tilde{\theta}^{(2)} \mid \tilde{\theta}^{(2)}<\theta^{*}<\tilde{\theta}^{(1)}\right]\right) \\
+\operatorname{Pr}\left[\tilde{\theta}^{(1)}<\theta^{*}\right]\left(0-E\left[\tilde{\theta}^{(2)} \mid \tilde{\theta}^{(1)}<\theta^{*}\right]\right) .
\end{gathered}
$$

We have $\operatorname{Pr}\left[\tilde{\theta}^{(2)}<\theta^{*}<\tilde{\theta}^{(1)}\right]=n F\left(\theta^{*}\right)^{n-1}\left(1-F\left(\theta^{*}\right)\right) \rightarrow 0$ as $\theta^{*} \rightarrow 1$. Thus, $R\left(\theta^{*}\right)<R(0)$ for all $\theta^{*}$ sufficiently close to 1 .

Let us now consider the case where $\theta^{*}$ is close to 0 . From $(76)$ it follows $\hat{b}_{s} \geq \hat{P}_{\theta^{*}}\left(\theta^{*}\right)$. Hence, (83) implies

$$
\begin{gathered}
R\left(\theta^{*}\right)-R(0) \geq \operatorname{Pr}\left[\tilde{\theta}^{(2)}<\theta^{*}<\tilde{\theta}^{(1)}\right]\left(\hat{P}_{\theta^{*}}\left(\theta^{*}\right)-E\left[\tilde{\theta}^{(2)} \mid \tilde{\theta}^{(2)}<\theta^{*}<\tilde{\theta}^{(1)}\right]\right) \\
-\operatorname{Pr}\left[\tilde{\theta}^{(1)}<\theta^{*}\right] E\left[\tilde{\theta}^{(2)} \mid \tilde{\theta}^{(1)}<\theta^{*}\right] .
\end{gathered}
$$


By Lemma 1 and because $\hat{r}^{\prime}(0)=1 / 2$,

$$
\begin{aligned}
\frac{\hat{P}_{\theta^{*}}\left(\theta^{*}\right)}{\theta^{*}} & =1-\frac{1}{\theta^{*} F\left(\theta^{*}\right)^{n-1}} \int_{\theta^{*} / 2}^{\theta^{*}}(f(0) \theta+\mathcal{o}(\theta))^{n-1} \mathrm{~d} \theta+\frac{\mathcal{o}\left(\theta^{*}\right)}{\theta^{*}} \\
& =1-\frac{1}{\theta^{*} F\left(\theta^{*}\right)^{n-1}} \int_{\theta^{*} / 2}^{\theta^{*}}\left(f(0)^{n-1} \theta^{n-1}+\mathcal{o}\left(\theta^{n-1}\right)\right) \mathrm{d} \theta+\frac{\mathcal{O}\left(\theta^{*}\right)}{\theta^{*}} \\
& =1-\frac{f(0)^{n-1}}{\theta^{*} F\left(\theta^{*}\right)^{n-1}}\left(\frac{\theta^{* n}}{n}\left(1-\frac{1}{2^{n}}\right)+\mathcal{o}\left(\theta^{* n}\right)\right)+\frac{\mathcal{o}\left(\theta^{*}\right)}{\theta^{*}}
\end{aligned}
$$

Therefore,

$$
\lim _{\theta^{*} \rightarrow 0} \frac{\hat{P}_{\theta^{*}}\left(\theta^{*}\right)}{\theta^{*}}=1-\frac{1}{n}\left(1-\frac{1}{2^{n}}\right)=\frac{n-1}{n}+\frac{1}{n 2^{n}}
$$

Similarly,

$$
\lim _{\theta^{*} \rightarrow 0} \frac{E\left[\tilde{\theta}^{(2)} \mid \tilde{\theta}^{(2)}<\theta^{*}<\tilde{\theta}^{(1)}\right]}{\theta^{*}}=\frac{n-1}{n}
$$

and

$$
\lim _{\theta^{*} \rightarrow 0} \frac{E\left[\tilde{\theta}^{(2)} \mid \tilde{\theta}^{(1)}<\theta^{*}\right]}{\theta^{*}}=\frac{n-1}{n+1}
$$

Moreover,

$$
\frac{\operatorname{Pr}\left[\tilde{\theta}^{(2)}<\theta^{*}<\tilde{\theta}^{(1)}\right]}{\operatorname{Pr}\left[\tilde{\theta}^{(1)}<\theta^{*}\right]}=n \frac{1-F\left(\theta^{*}\right)}{F\left(\theta^{*}\right)} \rightarrow_{\theta^{*} \rightarrow 0} \infty .
$$

Taking (84), (85), (86), (87), and (88) together implies that $R\left(\theta^{*}\right)>R(0)$ if $\theta^{*}$ is small. $Q E D$

The final Proposition 6 assumes that the initial seller is a player who's objective is to maximize expected revenue. We maintain the assumption that the initial seller is restricted to a second-price auction, but assume that she can set an arbitrary reserve price before the auction begins. Proposition 6 shows that the initial seller's revenue in such an extended second-price auction game with resale can be smaller than her revenue in the absence of a resale opportunity (where all bidders have a dominant strategy to bid their use values). ${ }^{11}$

Observe that a special case covered by Assumption 4 is that the resale seller is, like the initial seller, restricted to a second-price auction with reserve price. Hence, Proposition 6 shows that the presence of a resale opportunity can be harmful to the initial seller even if she has access to the same class of sales mechanisms as the resale seller.

\footnotetext{
${ }^{11}$ This result does not hold in environments with a single private-value bidder; cf. footnote 17 in the Note.
} 
Proposition 6 For any regular-bidder number $n \geq 2$ and distribution $F$ satisfying Assumption 1 , let $R^{*}$ denote the initial seller's revenue in a second-price auction when all bidders bid their use values and she chooses an optimal reserve price.

Then, for all sufficiently large $n$ and for all $\delta$ sufficiently close to 1 , the extended secondprice auction game with resale where the initial seller can set a reserve price has a perfect Bayesian equilibrium such that the initial seller's expected revenue is smaller than $R^{*}$.

PROOF. Let $r^{*}$ denote the reserve price that is optimal for the initial seller if all agents bid their use values in period 1 . We will show that if $\delta$ is sufficiently close to 1 , then there exists an equilibrium such that the initial seller sets a reserve price $r>r^{*}$ and her expected revenue is smaller than $R^{*}$.

Because $r^{*}<1$ is independent of $n$ (Myerson, 1981) and $R^{*} \rightarrow 1$ as $n \rightarrow \infty$,

$$
r^{*}<\delta R^{*}
$$

assuming that $n$ is sufficiently large and $\delta$ is sufficiently close to 1 .

Observe that the speculator's payoff in the $\theta^{*}$-equilibrium with $\theta^{*}=1$ constructed in Proposition 4 equals $\delta R^{*}$, and her auction bid $\hat{b}_{s}>R^{*}$ by (76) and Lemma 3. Hence, the 1-equilibrium remains valid, with a reduced payoff for the speculator, if the initial seller sets any reserve price $r<\delta R^{*}$.

By (89), there exists $\underline{r}$ such that

$$
r^{*}<\underline{r}<\delta R^{*}
$$

Using $\underline{r}$ we can construct continuation equilibria for the continuation games following any reserve price $r \geq 0$ by the initial seller. If $r<\underline{r}$, let the 1 -equilibrium be played in the continuation game. If $r>\underline{r}$, let the $\theta^{*}$-equilibrium with $\theta^{*}=0$ be played in the continuation game. If $r=\underline{r}$, let either the 1-equilibrium or the 0-equilibrium be played, depending on which of these two leads to a higher initial seller revenue.

Given these continuation equilibria, every reserve price $r<\underline{r}$ results in an expected revenue of $r<R^{*}$ for the initial seller. Any reserve price $r>\underline{r}$ leads to an initial seller revenue below $R^{*}$ because otherwise we would have $r=r^{*}$ by definition of $r^{*}$. The reserve 
price $r=\underline{r}$ leads to the initial seller revenue $\underline{r}<R^{*}$ if the 1-equilibrium is played, and leads to an initial seller revenue below $R^{*}$ if the 0 -equilibrium is played (if the revenue $R^{*}$ were reached, we would have $\underline{r}=r^{*}$ by definition of $r^{*}$ ). In summary, any reserve price $r \geq 0$ leads to an initial seller revenue below $R^{*}$.

\section{The English auction with resale}

In this section we explain why the equilibrium outcomes that we have constructed for secondprice auctions with resale remain valid if in period 1 an English auction, as modelled by Milgrom and Weber (1982), takes place. Observe that the second-price auction and the English auction are not strategically equivalent because we are considering environments with three or more bidders.

After the English auction is completed, the losers' bids are publicly known, while the winner's bid remains private. This corresponds exactly to the bid revelation that we have assumed in our analysis of the second-price auction with resale. Hence, if the bidders' stopping points in the English auction are identical to their bids in the second-price auction, the bidders' payoffs are the same across the two auction formats. Thus, the only thing we need to argue to show that the equilibrium outcomes of Proposition 4 remain valid for the English auction is that bidding incentives are the same as in a second-price auction. The difference between the second-price auction and the English auction is that the losing bids become public during instead of after the auction, so that bidders can revise their beliefs each time a bidder drops out.

We define bidding in the English auction as follows. Every bidder $i \in I$ with use value $\theta_{i} \in[0,1]$ stops bidding at price 0 if $\theta_{i}<\theta^{*}$, and is willing to bid up to her use value if $\theta_{i}>\theta^{*}$, independently of who stays in and how long. The speculator is willing to bid up to $\hat{b}_{s}$, also independently of who stays in and how long.

To see that these bidding strategies are optimal, consider first the speculator's bidding incentives, given the regular bidders' strategies. At the bid 0, it is better to stay in than to drop out because the latter means she foregoes her chances of winning and making a resale 
profit. If some regular bidder stays in beyond 0 as well, Bayesian updating requires the speculator to believe that the use value of this regular bidder is distributed on the support

$\left[\theta^{*}, 1\right]$. She expects the regular bidder to stay in up to her use value, which is beyond $\hat{b}_{s}$. Hence, it is optimal for the speculator to drop out at $\hat{b}_{s}$. If one regular bidder deviates by stopping at a bid in $\left(0, \theta^{*}\right]$, the speculator switches to the belief that the use value of this regular bidder equals $\theta^{*}$. Thus, if the speculator wins then she uses the same resale mechanism as in the second-price auction case.

Now consider the bidding incentives of a bidder $i \in I$ with use value $\theta_{i} \in[0,1]$. If $\theta_{i}<\theta^{*}$, bidder $i$ has no incentive to stay in beyond the bid 0 , for the same reasons as in the secondprice auction case. Finally, consider the case $\theta_{i}>\theta^{*}$. Bidder $i$ will stay in until the price reaches her use value because she expects the same from all other regular bidders once they stay in at positive bids. When bidder $i$ observes that a competing regular bidder drops out at a bid in $\left(0, \theta^{*}\right]$, she switches to the belief that this regular bidder has the use value $\theta^{*}$, and it remains optimal for bidder $i$ to stay in until the price reaches her use value.

\section{References}

Aubin, J.P., And A. Cellina (1984), Differential Inclusions, Springer Berlin.

Garratt, R., And T. Tröger (2005), Speculation in Standard Auctions with Resale, University of California at Santa Barbara and University of Bonn.

Haile, P. (1999), "Auctions With Resale," mimeo, University of Wisconsin, Madison.

Martinez, I. (2002), "First-Price Auctions where One Bidder's Value is Commonly Known," mimeo, University of Mannheim.

Milgrom, P., And R. Weber (1982), "A Theory of Auctions and Competitive Bidding," Econometrica 50, 1089-1122.

Myerson, R. (1981), "Optimal Auction Design," Mathematics of Operations Research 6, $58-73$.

Vickrey, W. (1961), "Counterspeculation, Auctions, and Competitive Sealed Tenders," Journal of Finance 16, 8-37. 